\title{
Spectroscopic evidence of grain ice mantle growth in YSOs
}

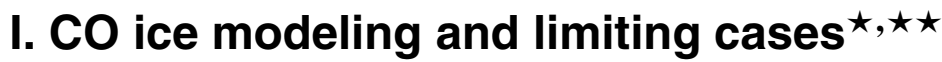

\author{
E. Dartois
}

Institut d'Astrophysique Spatiale, UMR 8617, Université Paris-Sud, bâtiment 121, 91405 Orsay, France

e-mail: emmanuel.dartois@ias.u-psud.fr

Received 14 April 2005 / Accepted 22 August 2005

\section{ABSTRACT}

\begin{abstract}
Using the Discrete Dipole Approximation for Scattering and Absorption of Light the volume attenuation coefficient of spherical and ellipsoidal coated grains is calculated, to simulate $\mathrm{CO}$ ice mantles on top of refractory grain cores in dense clouds. After estimating from observations and cosmic abundance constraints the expected mantle to core volume ratios, the simple analytical calculations that are generally used to model grain shape effects are discussed. It follows that the simple pure ice grain models are not applicable to the overall grain size distribution, but fairly accurately represent the smallest grains. Grain growth to micron sizes can reproduce the observed large red component in the CO absorption profiles toward many lines of sight, and traces the degree of scattering affecting the profile. Extreme case profiles such as the ones in the line-of-sight of CRBR 2422.8 - 3423 or Reipurth 50 are reproduced with such an approach. The continuum extraction made prior to analysing the ice profiles might be in some cases inadequate, as for micron-sized grains the influence of the extinction on the profile extends to several times the apparent pure absorption profile, modifying as a consequence their analysis.
\end{abstract}

Key words. dust, extinction - ISM: lines and bands - ISM: molecules

\section{Introduction}

In the relatively shielded environments provided by dense clouds, infrared astronomical observations have early revealed the presence of ice mantles covering grain "cores" composed of more refractory material (e.g. Gillett \& Forrest 1973; Merrill et al. 1976; Willner et al. 1982). These ices are of specific interest because they constitute an interface between a solid phase complex evolution under energetic processes and surface reactions with the rich chemistry taking place in the gas phase. Since the very first identifications, the assignments of the frozen molecules responsible for the observed features, mostly in absorption, have been based on the direct comparison of spectra of ice films produced at low temperature in the laboratory with astronomical observations (e.g. see articles referenced in the Dartois 2005; van Dishoeck 2004; Gibb et al. 2004, reviews). One exception is the long wavelength optical modes of water ice at about 44 and $66 \mu \mathrm{m}$ (e.g. Dartois et al. 1998). When ice mantles grow, in dense environments, some of the absorption features profiles start to deviate from pure laboratory transmittance spectra of ice films, due to the onset of grain size effects. The most commonly observed effect is the appearance of a long wavelength wing to the $3 \mu \mathrm{m}$

\footnotetext{
* Appendices A-D are only available in electronic form at http: //www. edpsciences.org

$\star \star$ Based on Experiments performed at IAS-CNRS and observations from ESO-VLT.
}

water ice stretching mode absorption band, when the grain size distribution upper sizes reach about $\lambda|m| / 2 \pi$ ( $\lambda$ is the wavelength and $m$ the complex refractive index of the material), i.e. $\sim 0.5$ in that case. This effect has been already reported by e.g. Smith et al. (1989). Deviation from the simple film transmittance spectra are often modeled with a continuous distribution of ellipsoids (CDE) of pure ice grains in the Rayleigh limit. Such a modeling assumes three hypothese that might be all violated in some astrophysical environments, where grains may either not be pure ice (ice constitute a mantle on top of or mixed with a refractory grain), or not in the Rayleigh limit. In addition, the CDE distribution used has no strong physically justified meaning as very large deviations from spheroidal shapes in collisional/accreting media with high turbulence remain to be demonstrated/observed.

In Sect. 2 we derive from astrophysical observations the expected range of mantle thicknesses from the ice to core volume ratio, the variation of thickness for individual grains pertaining to a typical size distribution and the limit in grain growth based only on accretion of atoms/molecules from the gas phase.

In Sect. 3, we will focus our attention on the $\mathrm{CO}$ ice profile as, due to its unique infrared active mode, it is probably one of the best ice test cases to see the ice growth effects. Historically, solid state CO observations (Lacy et al. 1984; Whittet et al. 1985; Geballe 1986) showed varying profiles decomposed using two underlying components, located at about $2135 \mathrm{~cm}^{-1}(\sim 4.68 \mu \mathrm{m})$ and $2140 \mathrm{~cm}^{-1}$ 
$(\sim 4.67 \mu \mathrm{m})$, with a full width at half maximum of about 10 and $3-5 \mathrm{~cm}^{-1}$, respectively. The presence of large amounts of $\mathrm{H}_{2} \mathrm{O}$ along these lines of sight led some authors to consider not only the pure $\mathrm{CO}$ ice case, which allows a good reproduction of the narrow component, but also the influence of the possible formation of an $\mathrm{H}_{2} \mathrm{O}-\mathrm{CO}$ hydrogen bonded complex (Sandford et al. 1988; Schmitt et al. 1989) on the strength and position of the unique $\mathrm{CO}$ vibration. Several authors extended the number of sources showing the variety of CO profiles (Mc Fadzean et al. 1989; Whittet et al. 1989; Eiroa \& Hodapp 1989; Kerr et al. 1991; ...). The narrow profiles of $\mathrm{CO}$ embedded in ice matrices made of $\mathrm{CO}, \mathrm{CO}_{2}$, $\mathrm{N}_{2}, \mathrm{O}_{2}$ and the larger ones when $\mathrm{CO}$ interacts with $\mathrm{H}_{2} \mathrm{O}$, $\mathrm{NH}_{3}, \mathrm{CH}_{3} \mathrm{OH}$ led to the separation of the two components into the so-called "non-polar" and "polar" CO profiles (Tielens et al. 1991). Since then has begun the search for minimizations, correlations and decompositions of astronomical $\mathrm{CO}$ profiles, to decipher the ice phases present along the lines of sights (e.g. Kerr et al. 1993; Palumbo \& Strazzulla 1993; Tanaka et al. 1994; Chiar et al. 1995; Chiar et al. 1998; Elsila et al. 1997; Teixeira et al. 1998; Boogert et al. 2002;...). A recent survey of low mass stars by Pontoppidan et al. (2003) confirmed the decomposition with the two previously cited components, plus a third blue one added for a full parametric decomposition.

The carbon monoxide ice unique vibrational absorption modeling presented in Sect. 3, from pure ice to silicate core coated spheres or ellipsoids, coupled to the grain growth to micron sizes, will give insight into the consequence of grain size and shape on the resulting line profile. We also explore briefly in Sect. 4 the multi-coated case and the carbonaceous core influence.

In Sect. 5, some contradictions arising under the "polar" "non polar" hypothesis for some CO profile fits found in the literature will be discussed together with astrophysical examples of particular relevance, as they can be considered as limiting cases. We will show that grain growth provides an alternative (complementary) way to reproduce the above cited red component or so-called "polar" feature observed in the $\mathrm{CO}$ absorption profiles toward many lines of sight.

This is followed by the conclusions in Sect. 6 .

\section{Constraints on astrophysical ice}

\subsection{Ice mantle growth}

Once inside a dense cloud with visual extinction above about three (the exact value depends on the particular cloud considered, e.g. Murakawa et al. 2000; Whittet et al. 1983), the refractory grains will acquire an ice mantle whose composition will depend on the species that accrete and react on the surfaces of these grains. To a first approximation, the volume increase of a grain can be described by the following steady state equation:

$4 \pi a^{2} \frac{\mathrm{d} a}{\mathrm{~d} t}=\pi a^{2} \sum_{i=1}^{N} n_{i} \frac{m_{i}}{\rho} v_{i} \eta$

where $a$ is the grain radius, $n_{i}$ and $m_{i}$ are the density and mass of impinging molecules or nucleon with velocity $v_{i}, \rho$ the mass volume density of the accreting mantle, $\eta$ is an "efficiency factor" which includes the sticking coefficient of the impactor, its reaction rate, etc, (the ratio $\eta m_{i} / \rho$ is the volume increase per impact). This equation is a stationary crude estimate of ice growth by accretion but already tells us that to first order $\mathrm{d} a / \mathrm{d} t$ is independent of the grain core radius. A more rigorous treatment can be found in e.g. Evans (1993), which lead to the same qualitative conclusion. We therefore assume hereafter that the mantle radius increase is a constant.

\subsection{Ice to silicates volume ratio}

Water ice optical depth has been observed to correlate with extinction along many lines of sight. This correlation appears after a visual extinction threshold has been reached, depending on the individual molecular clouds, and interpreted as due to the different degrees of clumping of the isolated dense clouds. In the case of the Taurus cloud, the correlation is well established (e.g. Murakawa et al. 2000; Whittet et al. 1983, and references therein), with:

$\tau\left(\mathrm{H}_{2} \mathrm{O}\right.$ ice $) \approx 0.067 H\left(A_{\mathrm{V}}-A_{\mathrm{V}}\right.$ threshold $)$

where $\tau\left(\mathrm{H}_{2} \mathrm{O}\right)$ is the optical depth in the $\mathrm{OH}$ stretching mode of water ice, $H$ is the Heaviside step function, $A_{\mathrm{V}}$ and $A_{\mathrm{V} \text { threshold }}$ are the visual extinction and ice visual appearance threshold along the line sight. If one add to this the relation found between silicates optical depth and visual extinction, $A_{\mathrm{V}} / \tau$ (silicates) $\approx 16.6$ (Rieke \& Lebofsky 1985) or more recently $A_{\mathrm{V}} / \tau$ (silicates) $\approx 19.2$ (Bowey et al. 2004), the following relation can be deduced: $\tau\left(\mathrm{H}_{2} \mathrm{O}\right.$ ice $) / \tau($ silicates $) \approx 1.1-1.3$. We can then evaluate the volume ratio using:

$\frac{V(\text { ice })}{V(\text { sil })}=\left(\frac{M_{\text {ice }}}{\rho_{\text {ice }}} N_{\mathrm{H}_{2} \mathrm{O}}\right) /\left(\frac{M_{\text {sil }}}{\rho_{\text {sil }}} \frac{N_{\mathrm{Si}}}{n_{\mathrm{Si}}}\right)$

with $N_{\mathrm{H}_{2} \mathrm{O}} \approx \frac{\tau_{\text {ice }} \Delta v_{\text {ice }}}{A_{\text {ice }}}$ and $N_{\mathrm{Si}} \approx \frac{\tau_{\text {sil }} \Delta v_{\text {sil }}}{A_{\text {sil }}}$

where $M_{\text {ice }}, \rho_{\text {ice }}, M_{\text {sil }}$, and $\rho_{\text {sil }}$ are the molar mass $(\mathrm{g} / \mathrm{mol})$ and density $\left(\mathrm{g} / \mathrm{cm}^{3}\right)$ for water ice and silicates, respectively. $N_{\mathrm{H}_{2} \mathrm{O}}$ and $N_{\mathrm{Si}}$ are the water molecules and silicon atoms in the observed silicates column density, respectively. $n_{\mathrm{Si}}$ is the number of silicon atoms in a mole of a given silicates. $\tau_{\text {ice }}, \Delta v_{\text {ice }}, A_{\text {ice }}$, $\tau_{\text {sil }}, \Delta v_{\text {sil }}, A_{\text {sil }}$, are the line center optical depth, line full width at half maximum $\left(\mathrm{cm}^{-1}\right)$ and integrated absorption cross section $(\mathrm{cm} /$ molecule $)$ of ice and silicates.

$M_{\text {sil }} /\left(\rho_{\text {sil }} n_{\mathrm{Si}}\right)$ is between about 30 and $40 \mathrm{~cm}^{3} / \mathrm{mol} / \mathrm{Si}$ for magnesium rich silicates (Reddy et al. 2004) and $M_{\mathrm{H}_{2} \mathrm{O}} / \rho_{\mathrm{H}_{2} \mathrm{O}}$ is about $19.6 \mathrm{~cm}^{3} / \mathrm{mol}$ for non-porous ice. Using $\Delta v_{\mathrm{H}_{2} \mathrm{O}}(3 \mu \mathrm{m}) \approx 300 \mathrm{~cm}^{-1}, A_{\mathrm{H}_{2} \mathrm{O}}(3 \mu \mathrm{m})=$ $2 \times 10^{-16} \mathrm{~cm} /$ molecule (D'Hendecourt \& Allamandola 1986; Gerakines et al. 1995) and $\Delta v_{\text {sil }}(10 \mu \mathrm{m}) \approx 300 \mathrm{~cm}^{-1}$, $A_{\text {sil }}(10 \mu \mathrm{m}) \approx 1.6-2 \times 10^{-16} \mathrm{~cm} /$ molecule, one can therefore deduce that at $A_{\mathrm{V}}$ well above $A_{V \text { threshold }}$ :

$f_{\text {vol }}=\frac{V(\text { ice })}{V(\text { sil })} \gtrsim 0.5-0.8$.

In practice, this value establishes only a lower limit to the actual ice-to-refractory mantle volume ratio, as one should include 


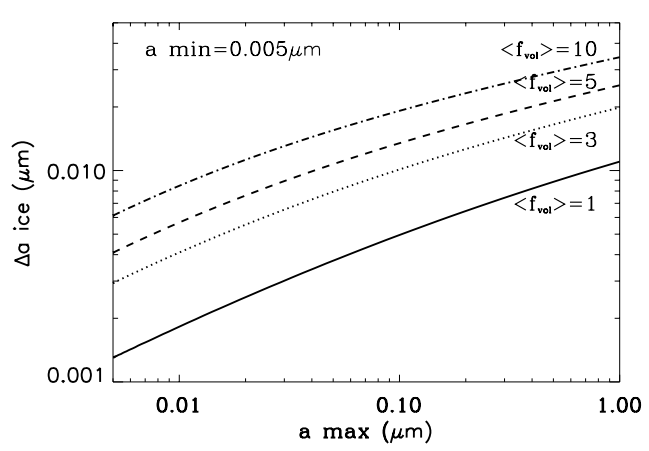

Fig. 1. Ice mantle thickness $\Delta a$ in an MRN dust grain distribution with fixed minimum size of $a_{-}=0.005 \mu \mathrm{m}$ and variable upper size $a_{+}$, for a total mean ice mantle to core volume ratio $f_{\mathrm{vol}}=\{1,3,5,10\}$.

all ices and recall that one integrates the silicate optical depth along lines of sight where some of the grains are uncoated (in particular where the grain temperatures are above mantle evaporations, near protostellar objects). We will therefore explore the $f_{\mathrm{vol}}=1-10$ domain.

\subsection{Grain size distribution}

The ice-to-silicate volume ratio was evaluated in the previous section. The mantle radius $\Delta a$ is a constant that one must estimate before calculation. Assuming that grains entering a dense cloud before the formation of ice mantles follow a classical MRN size distribution (Mathis et al. 1977), i.e. that the distribution of grains radii " $a$ " follow a power law $\left(n(a) \mathrm{d} a=a^{-3.5}\right)$, the total volume of silicates and ice are given by:

$f_{\mathrm{vol}}=\frac{V(\text { ice })}{V(\text { sil })}=\frac{\int_{a_{-}}^{a_{+}} \frac{4}{3} \pi(a+\Delta a)^{3} a^{-3.5} \mathrm{~d} a}{\int_{a_{-}}^{a_{+}} \frac{4}{3} \pi a^{3} a^{-3.5} \mathrm{~d} a}-1$

where $a_{-}$and $a_{+}$are the minimum and maximum size of grains in the MRN distribution. The above equations lead to:

$$
\begin{aligned}
2 f_{\mathrm{vol}}\left(\sqrt{a_{+}}-\sqrt{a_{-}}\right)=\frac{a_{-}^{-2.5}-a_{+}^{-2.5}}{2.5} \Delta a^{3} & +2\left(a_{-}^{-1.5}-a_{+}^{-1.5}\right) \Delta a^{2} \\
& +6\left(a_{-}^{-0.5}-a_{+}^{-0.5}\right) \Delta a .
\end{aligned}
$$

Figure 1 displays the calculated $\Delta a$ in an MRN distribution for a given $f_{\mathrm{vol}}$, fixed minimum size $\left(a_{-}\right)$and variable maximum size $\left(a_{+}\right)$. The corresponding individual volume fraction by size are given in Fig. 2.

\subsection{Grain growth by coagulation}

Given that the grain sizes will increase, they cannot attain several micron size only by accreting ice mantles. They must grow at the expense of the modification of the initial size distribution. This result proceeds from abundance constraints on the atoms participating in the mantle formation. Indeed, about $10 \%$ of the cosmic oxygen is locked into the ice mantles in dense clouds and if one assumes that it could rise to $100 \%$, the thickness of the mantle would increase by at most a few times its actual value, far from the growth expected in very dense circumstellar environments. This will have consequences on the way we

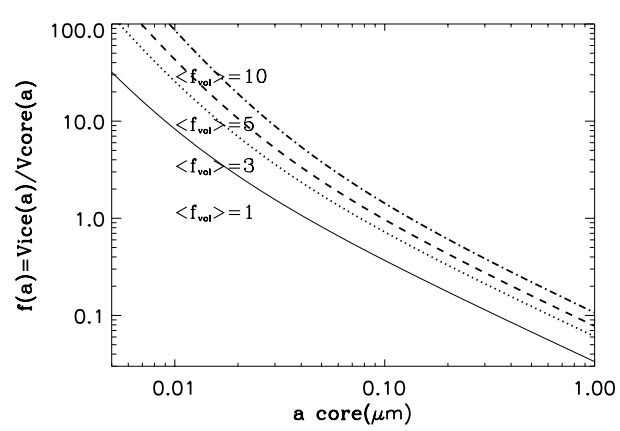

Fig. 2. True volume ratio for each individual grain size as a function of total mean ice mantle to core volume ratio $f_{\mathrm{vol}}=\{1,3,5,10\}$ for an MRN distribution with lower and upper bounds of $a_{-}=0.005 \mu \mathrm{m}$ and $a_{+}=1 \mu \mathrm{m}$.

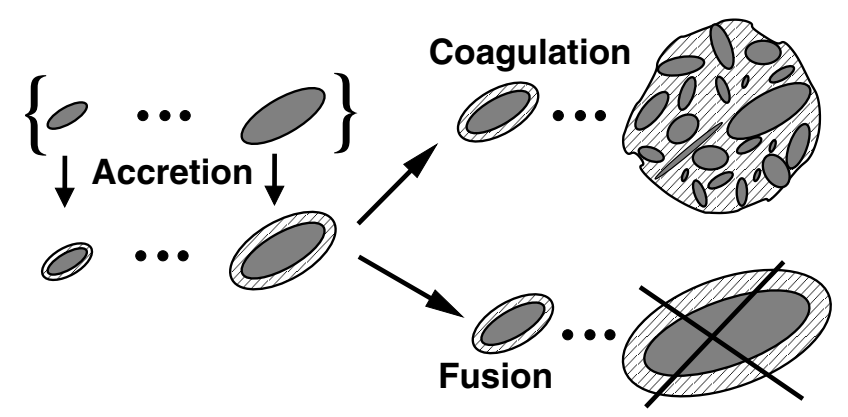

Fig. 3. Schematic representation of the grain growth in dense clouds. Coagulation of small grains should lead to the formation of heterogeneous mixed ice/silicates grains and not the idealised fusion of the cores into a unique grain covered by a mantle.

model large grains made of ice mixed with refractory elements. As illustrated in Fig. 3, the process of coagulation should not produce "fused" large homogeneous grains composed of a single core and mantle, but rather an heterogeneous coagulated ensemble of small to intermediate core/mantles, at least in the first phases of dense cloud coagulation where temperatures leading to the fusion of the cores is not sufficient.

\subsection{Modification of the size distribution by coagulation}

The total grain volume in an initial power law distribution of grain follows:

$V_{\mathrm{i}}(\mathrm{tot}) \propto \int_{a_{\mathrm{i}-}}^{a_{\mathrm{i}+}} \frac{4}{3} \pi a^{3} a^{n} \mathrm{~d} a \propto a_{\mathrm{i}+}^{4+n}-a_{\mathrm{i}-}^{4+n}$

where $n$ is the index of the law ( $n=-3.5$ for MRN). Let assume the total volume of matter is conserved, within the limits imposed by the above mentioned accretion, and that the most probable accretions will first proceed through the coagulation of small particles with others. Therefore, by sweeping off these small ones from the distribution to the benefit of the large ones, a first order estimate of the new size distribution limits is given by:

$\frac{V_{\mathrm{f}}(\mathrm{tot})}{V_{\mathrm{i}}(\mathrm{tot})}=1=\frac{a_{\mathrm{f}+}^{4+n}-a_{\mathrm{f}-}^{4+n}}{a_{\mathrm{i}+}^{4+n}-a_{\mathrm{i}-}^{4+n}}$

$a_{f-}=\left(a_{\mathrm{i}-}^{4+n}-a_{\mathrm{i}+}^{4+n}+a_{\mathrm{f}+}^{4+n}\right)^{1 /(4+n)}$. 


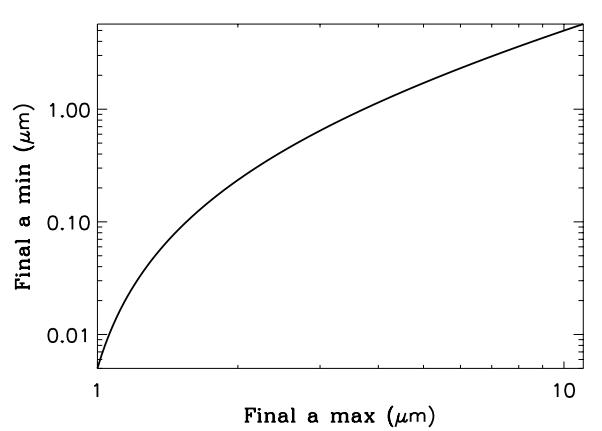

Fig. 4. Representation of the lower size limit $a_{-}$of an MRN-like power law distribution as a function of the upper limit $a_{+}$, at fixed total volume set by initial MRN parameters $a_{-}=0.005 \mu \mathrm{m}$ and $a_{+}=1 \mu \mathrm{m}$.

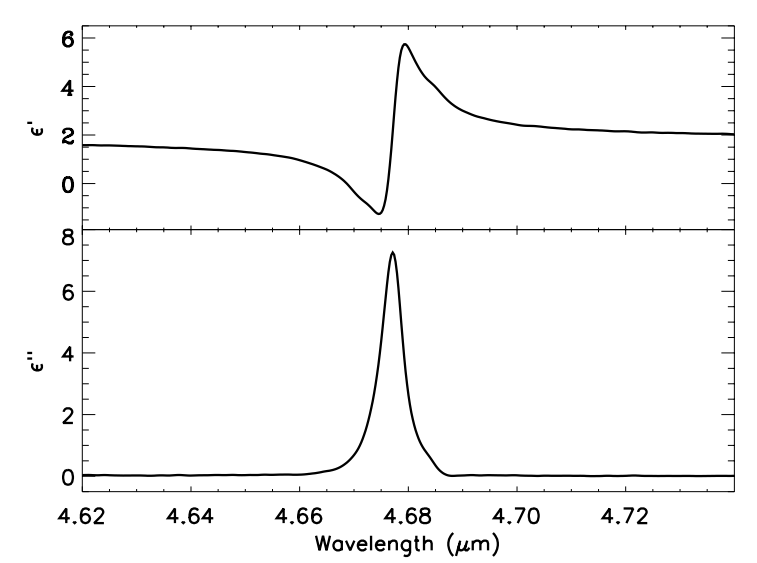

Fig. 5. Adopted CO dielectric function. They have been determined experimentally from transmittance spectra of pure CO films deposited at 10 K. See also Baratta \& Palumbo (1998) and Elsila et al. (1997).

This behaviour is illustrated in Fig. 4. For a moderate increase of the upper size of the distribution, the lower size cut-off increases substantially. Such a shift of the distribution limits will have important consequences for the resulting profile of the ices.

\section{Modeling of line profiles}

\subsection{Adopted optical constants}

We now focus on the assumptions of the grain shapes generally used to calculate and reproduce the line profile of ice features.

The best ice test case is the carbon monoxide line profile. $\mathrm{CO}$ is observable at high resolution from the ground in a single setup. It is one of the first profiles that should be affected by the grain growth as size parameters of unity are reached with smaller grain size at the shortest wavelengths. In addition, $\mathrm{CO}$ should not present too complex profiles, even when in intermolecular interactions in a matrix, as a diatomic molecule. We choose in a first step to use pure CO optical constants to deduce the effects of scattering on line profiles in the absence of ice intermolecular interactions. The dielectric functions adopted for CO are given in Fig. 5. They have been determined experimentally from transmittance spectra recorded at normal incidence on pure $\mathrm{CO}$ films produced at $10 \mathrm{~K}$ at the Institut d'Astrophysique Spatiale at a spectral resolution of $0.25 \mathrm{~cm}^{-1}$.

To achieve this, several transmittance spectra of thin films of a fraction of a micron to a few microns thickness were acquired on our IFS $66 \mathrm{~V}$ Bruker spectrometer coupled to a cryostat cooled to $10 \mathrm{~K}$ with liquid helium. These spectra were modeled using classical transmission equations of a thin film deposited on a thick transparent substrate, as in e.g. Swanepoel (1983). This modeling was combined with an iterative scheme which progressively refines the refractive index $n$, to model the next step based on modified Kramers-Kronig relations (e.g. Trotta 1996, and reference therein) applied to the imaginary part $k$ of the refractive index. Isotopes absorptions $\left({ }^{13} \mathrm{CO}\right)$ were used to check for the relative thickness scaling of each spectrum. This is made possible because ${ }^{13} \mathrm{CO}$ is a much less abundant species in the ice formed and therefore does not suffer a large deviation from a pure absorption spectrum given that the refractive index $\mathrm{n}$ stays almost constant along the feature. Equations relating the refractive index to the dielectric functions ${ }^{1}$ are then used. The deduced $\epsilon^{\prime}$ and $\epsilon^{\prime \prime}$ are in close agreement with the ones found in Baratta \& Palumbo (1998) and Elsila et al. (1997), although these authors performed their analysis at lower resolving power $\left(2 \mathrm{~cm}^{-1}\right.$ and $1 \mathrm{~cm}^{-1}$, respectively). This holds provided the dielectric functions are fitted with a Lorentz oscillator model (See Fig. 15 of Pontoppidan et al. 2003). The Full Width at Half Maximum (FWHM) of $\epsilon^{\prime \prime}$ for pure $\mathrm{CO}$ being above $2 \mathrm{~cm}^{-1}$, the achieved resolution adopted here is sufficient to well sample this band.

When we treat ice as a mantle, the composition of the core is based on the observed amorphous silicates whose mean dielectric constants adopted are the Draine \& Lee ones (Draine \& Lee 1984).

Much more sophisticated constants including carbonaceous material also could have been used, but the corresponding exact dielectric function are, from the astrophysical point of view, still unconstrained or poorly constrained in the infrared. A good example is that it is still under debate whether the carbonaceous fraction is covering silicates or a separate phase. Observations have been performed on the subject using polarimetry (Adamson et al. 1999), but at present are still controversial in the field. This will not affect our global reasoning nor our demonstration on ice profiles, but it would be necessary to include them as soon as they are better constrained.

\subsection{From pure ice to coated sphere}

The simplest ice grain is a pure sphere of ice. Real constraints imply a core-mantle structure, as ice mantles grow on existing refractory grains that were present before entering the dense cloud phase. In the Rayleigh regime, where the size parameter $x$ is much lower than unity (where $x=2 \pi a / \lambda, a$ radius of the grain, $\lambda$ the wavelength), the extinction cross section of such a grain can be described analytically, and are given both for a sphere and a coated sphere in Appendix A. When the size parameter becomes of the same order of

\footnotetext{
1 These relations are given by $\epsilon^{\prime}=n^{2}-k^{2}, \epsilon^{\prime \prime}=2 n k$, and, conversely $n=\sqrt{\left[\left(\epsilon^{\prime 2}+\epsilon^{\prime \prime 2}\right)^{1 / 2}+\epsilon^{\prime}\right] / 2}, k=\sqrt{\left[\left(\epsilon^{\prime 2}+\epsilon^{\prime 2}\right)^{1 / 2}-\epsilon^{\prime}\right] / 2}$.
} 

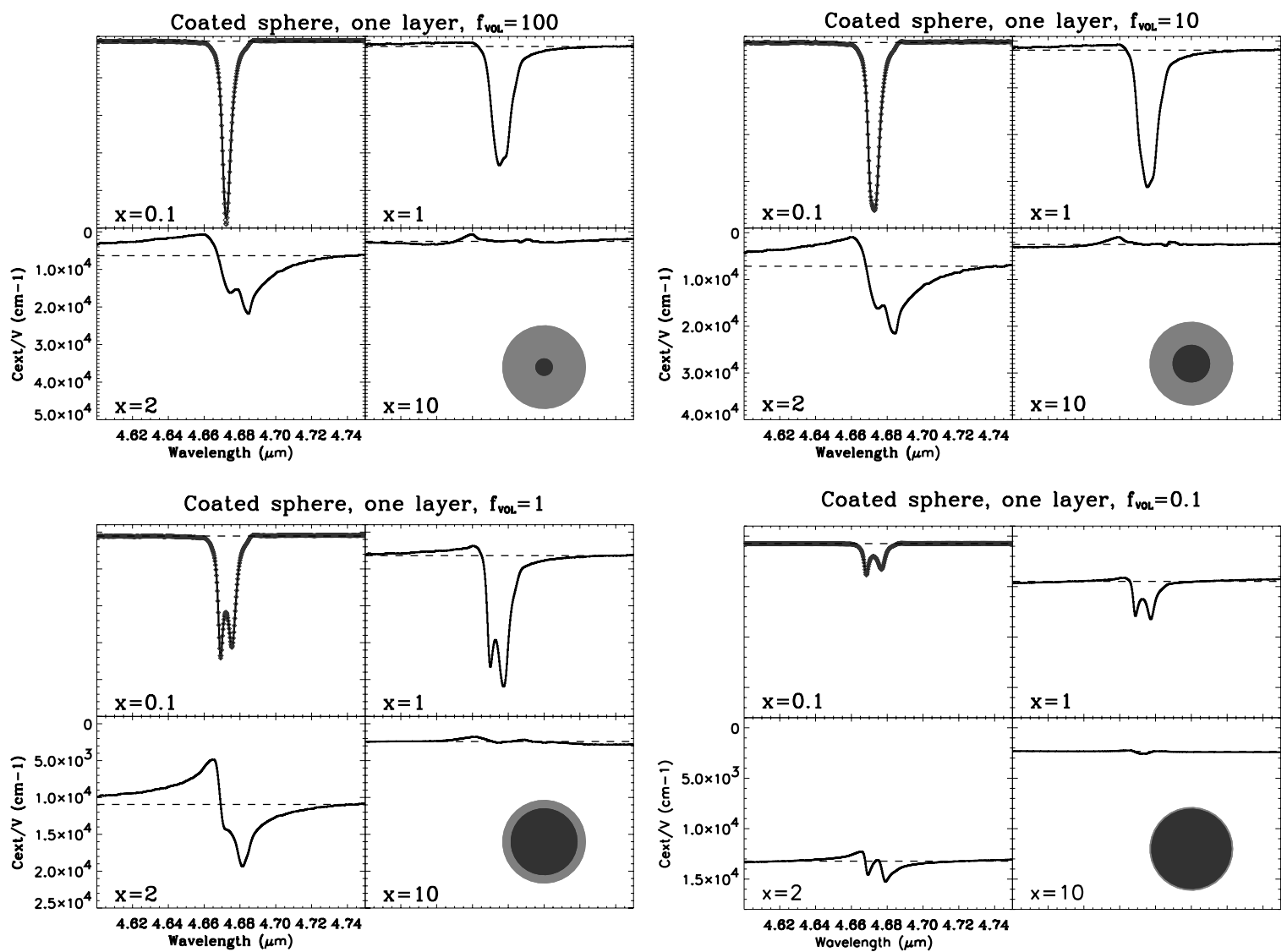

Fig. 6. Volume attenuation coefficient of coated spheres with mantle (CO ice) to core (silicates) volume ratio ranging from 100 to 0.1 . In each panel, corresponding to a given volume ratio, calculations are presented for representative size parameters of $0.1,1,2,10$. A schematic drawing representing the physical volume of the silicates core (black) and ice mantle (grey) is inserted in each panel.

or larger than unity, a full treatment must be performed to solve the field equations. Algorithms have been developed to calculate the resulting far field cross-sections for coated and multi-coated spheres (e.g. Toon \& Ackerman 1981, bhcoat from Bohren \& Huffman 1983; Bhandari 1985; Kai \& Massoli 1994; Wu et al. 1997). Many of them are valid only for size parameters up to a few times unity, and especially fail in the case of non-absorbing or strongly absorbing spheres. A recent recast by Yang (2003) of the formula used in such algorithms, allowing the calculation of multi-layered spheres, has proved to be accurate and valid at large size parameters without accumulating numerical instabilities in the recurrence schemes intrinsic to these calculations. We use this scheme, summarised in Appendix B, for the general case of coated spheres. When applicable $(x \ll 1)$, we also compare the results to analytical expressions.

We calculate the volume attenuation coefficient $\alpha_{\mathrm{V}}=$ $C_{\text {ext }} / V$ for four representative size parameters to describe the effects on the cross-sections of the increase in size parameter. We start from the end of the Rayleigh regime $(x \approx 0.1)$, go through the scattering regime $(x \approx\{1,2\})$ and end at the very beginning of geometrical optics ( $x \approx 10)$. These size parameter values are approximate, as they are defined strictly, in our calculations, for a wavelength of $4.67 \mu \mathrm{m}$ as we work for each calculation with a fixed grain radius for obvious physical reasons.
In a power law size distribution such as the MRN one, with an index $n=-3.5$ the weighting that must be applied to individual grains to calculate the mean extinction of the overall distribution is proportional to $\alpha_{V} \times a^{-0.5}$. This is sufficiently close to uniform weighting to allow direct meaningful comparisons between $\alpha_{V}$ for different grain sizes to estimate the effects of individual grains on the final profile for such a distribution. This behaviour motivated us to use the $\alpha_{V}$ representation of cross-sections. To derive the mass attenuation coefficient $\alpha_{M}$, one must divide $\alpha_{V}$ by the mean density $\langle\rho\rangle$ of the particle.

$\alpha_{V}$ calculations for coated spheres with $x \approx\{0.1,1,2,10\}$ and $f_{\mathrm{vol}}=\{100,10,1,0.1\}$ are presented in Fig. 6 , ordered by decreasing ice volume fraction. When $x \approx 0.1$, we have superimposed (small diamond) the analytical calculations given in Appendix A in the Rayleigh limit for a pure $\mathrm{CO}$ sphere in the case $f_{\mathrm{vol}}=100$, and a coated sphere for $f_{\mathrm{vol}}=\{10,1,0.1\}$. The agreement in the asymptotic behaviour is excellent and the case $f_{\mathrm{vol}}=100$ can be considered as almost equal to the pure ice sphere case.

\subsection{Information from the coated sphere ideal case}

Even with a simple grain model like the coated sphere one, we can deduce important factors. 
The CO feature absorption width is dependent on the $f_{\text {vol }}$ ratio, even in the Rayleigh regime. Therefore, assuming pure ice spheres to represent true astrophysical grain analogs is not valid in a second order analysis.

The presence of a core with a refractive index different from the ice mantle induces the apparition of two peaks when the volume ratio of ice to silicates is less than about a few units. In a MRN distribution, this can be counterbalanced to a certain degree by the fact that small grains will absorb more efficiently and that they are the ones with the highest $f_{\text {vol }}$ ratio (see Fig. 2)

Increasing the size parameter, i.e. the grain sizes at fixed wavelength, brings strong asymmetries in the profile of the CO ice band, whatever the $f_{\text {vol }}$ ratio. The asymmetry develops following roughly the behaviour of $\epsilon^{\prime}$.

The very big grains (with respect to the observed wavelength) contribute to a grey opacity. Thus, no noticeable features can be observed from their presence in a distribution containing large amounts of small grains. Grains much larger than the wavelength will only contribute to a continuum modification, not a feature profile modification. In other words, the presence of these grains is not well constrained by the shape of an absorption feature, unless the continuum can be understood as an absolute value.

As a consequence of different size contributions in a distribution, small grains dominate the absorption centre of the line profile whereas the intermediate grains will contribute to asymmetries in the wings.

\subsection{From pure ice to coated ellipsoids}

The next step in the calculations assumes ellipsoidal rather than spherical shape for the grains. The ellipsoid case is supported by the linear polarisation observed in extinction in the visible which suggest there is a dichroic absorption of nonspherical grains aligned with galactic magnetic fields (e.g. Scarrott et al. 1990). For ellipsoids, the orientation of the grain with respect to the incoming light has consequences on the extinction cross-section. We therefore calculate the average absorption cross-section of randomly oriented ellipsoids and take into account a distribution of all possible ellipsoid shapes, weighted by the probability of finding a given arbitrary shape in the distribution.

The simplest approach consists of modeling the grain distribution using a uniform weighting probability function in the Rayleigh limit. This give rise to the uniform Continuous Distribution of Ellipsoids, the so-called CDE, whose analytical cross section is given in Appendix A. In practice this assumption is physically unjustified as it implies an infinitely long and thin cylindric-like ellipsoid has the same probability of occuring as a spherical or close to sphere shaped one. In the absence of external constraints on the shape probability function behaviour, it is an ad-hoc hypothesis and mathematical fitting procedure. It is preferable and certainly judicious to use a weighting function favouring spheroidal shapes. The quadratic weighting function that discards extreme shapes gives rise to the Weighted Continuous Distribution of
Ellipsoids named WCDE in the Appendix A, and used e.g. in Fabian et al. (2001).

Out of the Rayleigh limit, there are no simple analytical representations of the cross-section for arbitrary non-confocal coated ellipsoids. Computational approaches based on the separation of variables method (SVM), T-Matrix method (TMM) and discrete dipole approximation (DDA) methods are then often employed to numerically solve the scattering problem. We make use in our case of the DDA program DDSCAT ${ }^{2}$ (Draine \& Flatau 2003).

Calculations were performed over all possible relative nondegenerated integer aspect ratios of the three axes $a, b, c$ between 1 and 5, having fixed the first axis to the highest integer (15 possibilities in total), weighted by the quadratic shape probability function.

The average of calculated extinctions for 16 orientations with respect to the incoming light, for the defined ellipsoids in the aspect ratio distribution, gives rise to the final volume attenuation coefficient presented in Fig. 7. More explanations are given in Appendix B. The number of discrete dipoles used in the calculations ranged from 5000 up to 35000 . These numbers were defined and constrained by the numerical precision needed at large size parameters, but also by the thinness of the coating requiring a large number of dipoles to be correctly described. To avoid prohibitive time calculations based on these constraints, we did not calculate the $f_{\text {vol }}=0.1$ series as in the coated sphere case. In addition to the coated ellipsoids the volume attenuation coefficient of ellipsoids with a dielectric function calculated using the Bruggeman or Looyenga effective medium theory (EMT, see Appendix D) is displayed in the $f_{\mathrm{vol}}=1$ case, for comparison.

\subsection{Information from the coated ellipsoids ideal case}

It is readily observed in Fig.7 that a continuous distribution of ellipsoids gives rise to wider absorption widths than spheres at equivalent size parameters. This is a consequence of the distribution of shapes broadening absorption bands (see e.g. Bohren \& Huffman 1983). The corresponding peak maximum absorption is reduced.

The major characteristics already observed for spheres when increasing the size parameter, i.e. the grain sizes, are similar: a strong asymmetry in the profile whatever the $f_{\text {vol }}$ ratio which develops following roughly the behaviour of $\epsilon^{\prime}$. In addition, the substructures are broadened and merge within the profile.

When comparing the coated case to the EMT calculations, the same global shape behaviour is obtained, but the small substructures inherent to the resonances of a well defined ice to silicates interface have disappeared. The EMT case should be more representative of the largest grains in the MRN distribution, following the coagulation process in the early phases of cloud condensations (see Fig. 3).

\footnotetext{
2 A description of the program can be found at http://wwW . astro.princeton. edu/ draine
} 

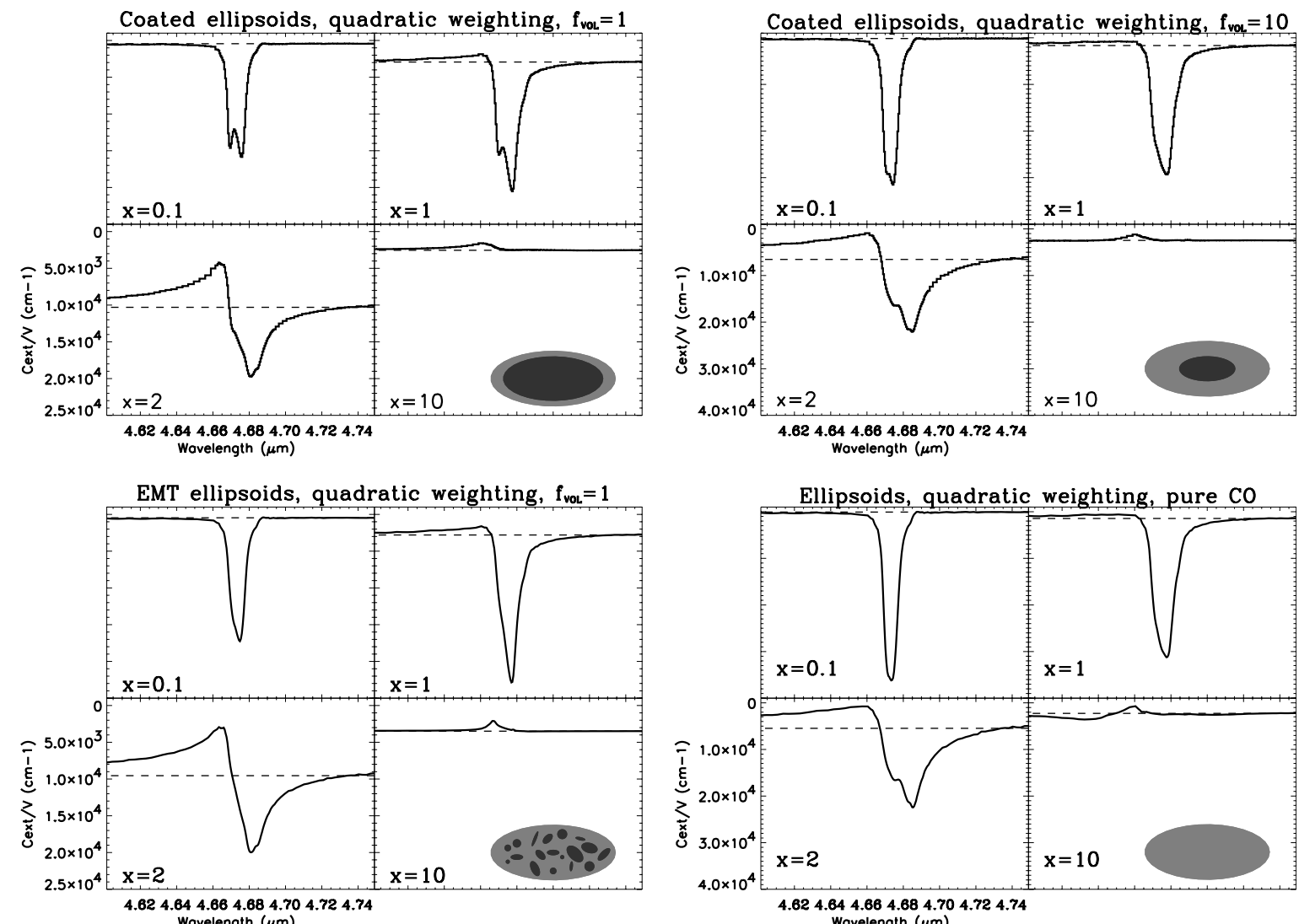

Fig. 7. Volume attenuation coefficient of a quadratic weighting continuous distribution of coated ellipsoids with mantle (CO ice) to core (silicates) volume ratio ranging from 1 to $\infty$ (see text and Appendix B for details). In addition, the calculation using the Bruggeman or Looyenga effective medium theory (EMT) for a volume ratio of 1 is also presented below the corresponding coated case. In each panel, corresponding to a given volume ratio, calculations are presented for representative size parameters of $0.1,1,2,10$. A schematic drawing of the physical volume of the core (black) and mantle (grey) for a representative ellipsoid with axes ratios $a, b, c=2,1,1$ is inserted in each panel for clarity.
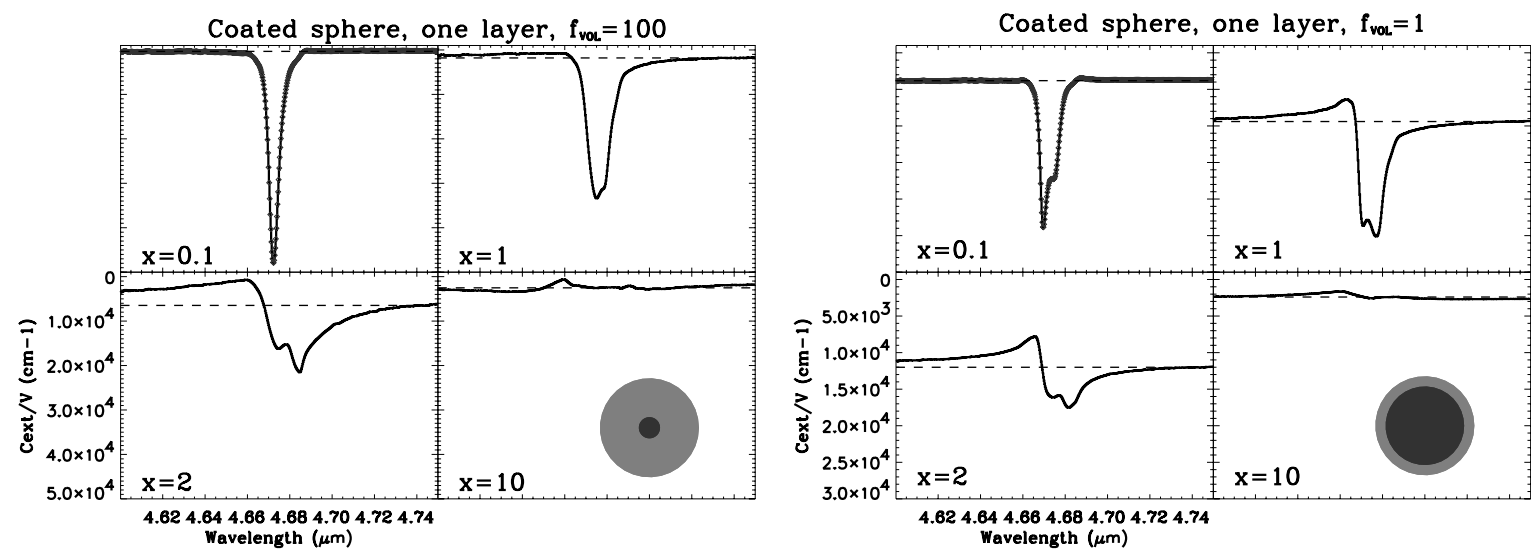

Fig. 8. Volume attenuation coefficient of coated spheres with mantle (CO ice) to carbonaceous core $\left(\mathrm{ACH}_{2}\right)$ volume ratio of 100 and 1 and size parameters ranging from 0.1 to 10 .

\section{Other examples}

In space one could expect to find onion-like mantles, with the more refractory material inside (e.g. water ice) and the more volatile outside (e.g. CO ice). In addition, the core of some of the grains can be composed of a material other than silicates, like a carbonaceous one such as a-C:H (or HAC). We therefore present two examples calculated under such conditions.

\subsection{Carbonaceous core/CO ice}

In Fig. 8 we present the calculation for a carbonaceous core $\left(\mathrm{ACH}_{2}\right.$, whose complex refractive index was obtained from Zubko et al. 1996) coated with CO ice, in the case where the volume ratio is 100 and 1 . It can be compared with the same calculations presented in Fig. 6 . In the $f_{\mathrm{vol}}=100$ case, the results are almost the same, indicating that the electro-magnetic wave does not feel the presence of the seed core and that it 

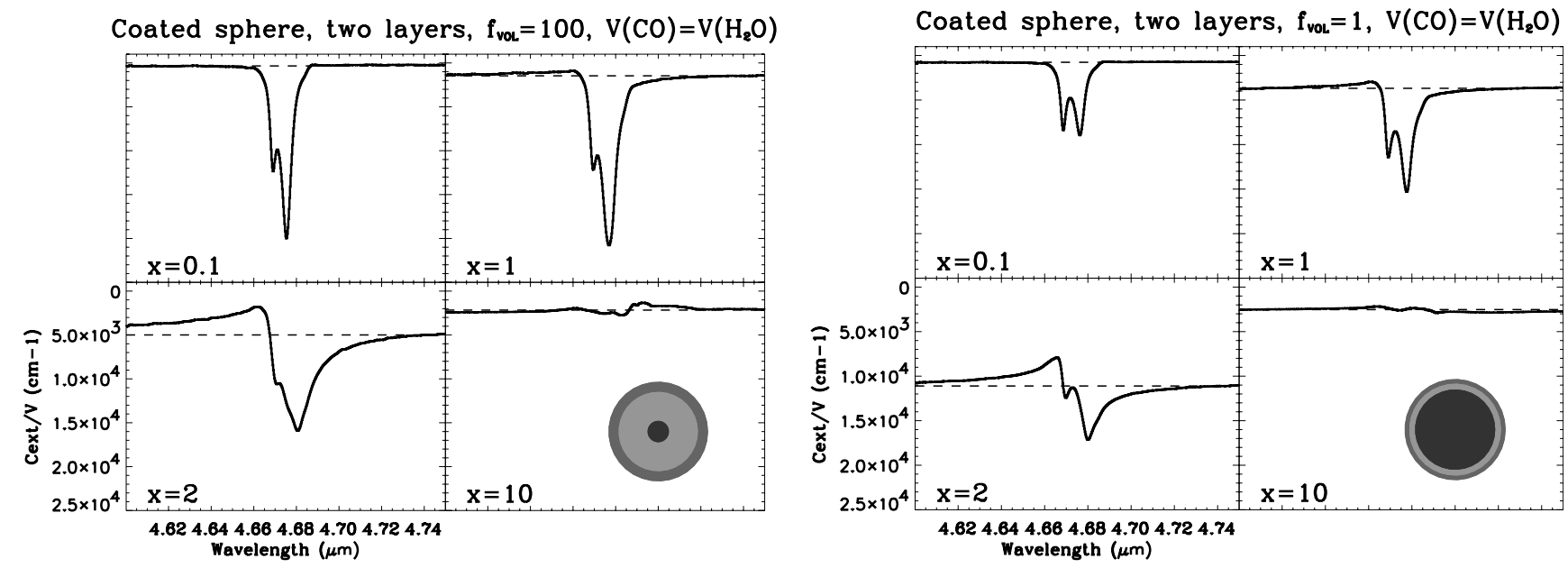

Fig. 9. Volume attenuation coefficient of doubly coated spheres with mantle $\left(\mathrm{CO}+\mathrm{H}_{2} \mathrm{O}\right.$ ice) to core (silicates) volume ratio ranging from 100 to 0.1 . The volume fraction of $\mathrm{CO}$ with respect to $\mathrm{H}_{2} \mathrm{O}$ is kept constant at 1 .

is equivalent to the pure ice case. The situation changes when $f_{\text {vol }}=1$. The pronounced two peak feature seen with the silicate core (in Fig. 6) is here reduced when replaced by the $\mathrm{ACH}_{2}$ one, as a consequence of a higher imaginary index of the latter compared to the former. The carbonaceous amorphous core acts as a damping parameter for the incoming wave.

\subsection{Multi-coated silicates/water ice/ CO ice}

In Fig. 9 we present the calculations for a two layer ice mantle. The calculations were performed using the multilayer algorithm developed by Yang (2003), and are detailed in Appendix C, with a water mantle (dielectric function adopted from Trotta 1996) in between a silicate core and an outer CO mantle. A constant $\mathrm{H}_{2} \mathrm{O}$ to $\mathrm{CO}$ ratio of 1 was fixed. In the $f_{\mathrm{vol}}=100$ case, the feature displays two peaks, showing that it is affected by the presence of the underlying water ice mantle refractive index that stays almost constant along the wavelength range. The effect is similar but less pronounced due to a lower refractive index than the one with a silicate core of the size of the water ice mantle. The effects are stronger with $f_{\text {vol }}=1$, where both the core and the water ice mantle influence the line shape of the CO absorption.

Apart from these material dependent effects, the same trend with size is observed as the one encountered in the previous sections, i.e. a warping when $x$ approaches or is just above unity.

\subsection{Some effects of porosity}

Interstellar grains may not be compact and the porosity might affect the observed line shapes. To gain insight into the influence of porosity, we modeled an ellipsoid coated by a $\mathrm{CO}$ ice layer with a porosity of $50 \%$. The calculations were performed using the DDSCAT program and the optical constants of the layer determined using the Looyenga effective medium theory. The same result was obtained with the Bruggeman theory for the mixture CO:vacuum. We did not employ the MaxwellGarnett EMT because of its lack of symmetry with respect to the inclusion and the matrix, which makes it by definition inappropriate for materials whose proportions are similar in a matrix. The details of how to calculate these EMT dielectric functions are given in Appendix D. The effect of vacuum on the optical constants is to reduce the mean refractive index of the transition as well as its amplitude. The index contrasts are lowered and the consequence, as seen in Fig. 10, is to smear out the substructures in the absorption profile.

\section{Astrophysical profiles}

The recent $M$ band (4.3-5 $\mu \mathrm{m})$ study of CO ice observed at moderate to high resolution with the VLT along many low mass young stellar object lines of sight (Pontoppidan et al. 2003) has allowed us to obtain good statistics on individual CO line profiles. The CO optical depth profiles were extracted by fitting a local blackbody continuum going through the $4.52-4.55 \mu \mathrm{m}$ and $4.76-4.8 \mu \mathrm{m}$ wavelength intervals. The profiles were then analysed using a phenomenological decomposition with the principal and strongest absorption centred at $2139.9 \mathrm{~cm}^{-1}(4.673 \mu \mathrm{m})$. Two additional Gaussian components, a blue $\left(2143.7 \mathrm{~cm}^{-1}\right)$ and a red one $\left(2136.5 \mathrm{~cm}^{-1}\right)$, were necessary and were added to allow a complete description of the profiles. The central peak is compatible with (and can readily be attributed to) small ellipsoidal grains made of almost pure $\mathrm{CO}$ or with large $\mathrm{CO}$ mantles such as the ones presented in Fig. 7 with $f_{\text {vol }}=\infty$ or $f_{\text {vol }}=10$. The red Gaussian required to provide a good fit to the profile is much wider $\left(10.6 \mathrm{~cm}^{-1}\right)$ than the central component $\left(3.5 \mathrm{~cm}^{-1}\right)$. This component is generally attributed to $\mathrm{CO}$ embedded in a water matrix, and before using it over astronomical spectra the grain size effects presented above, we investigate the assignment of the red component.

In most of the fits to the observed astronomical CO profile in the literature, the search for the best decomposition is performed using a peak position versus full width at half maximum (FWHM) diagram of CO ices in various matrices, in some cases including temperature variations.

Other important parameters such as the coherence in terms of abundance (or column density) constraints or stability of the 

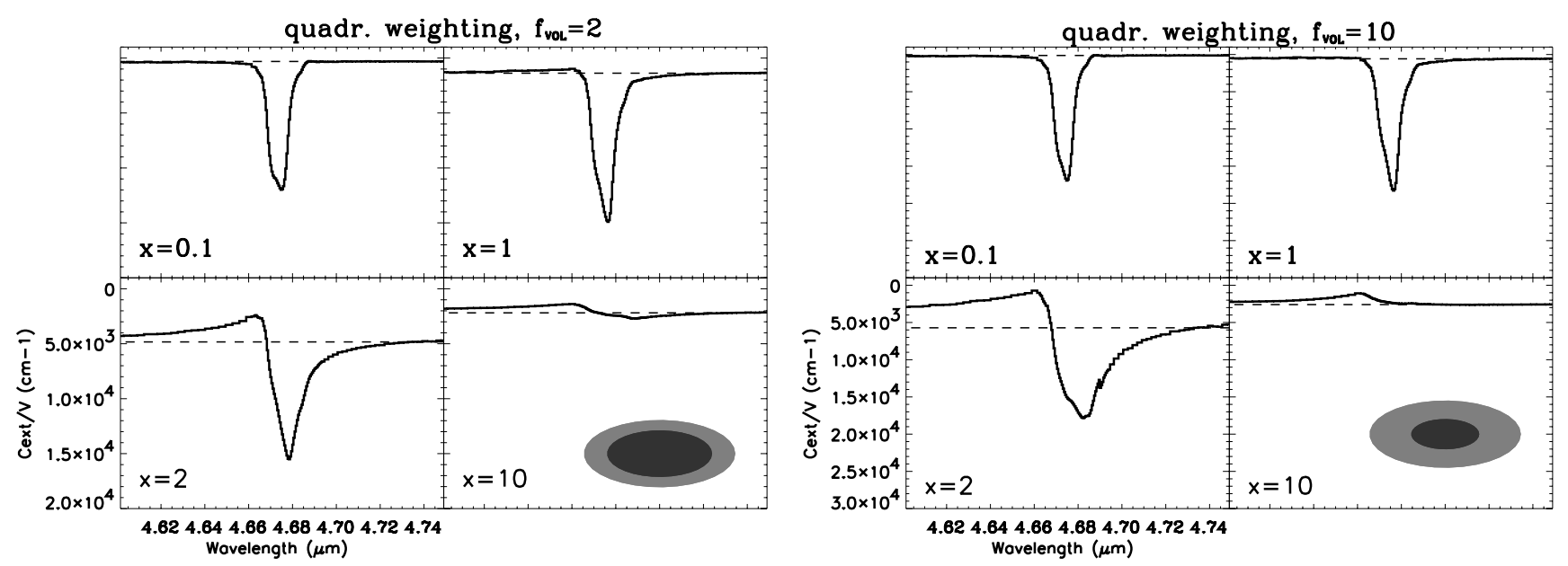

Fig. 10. Volume attenuation coefficient of coated ellipsoids coated with mantle (50\% $\mathrm{CO}$ ice; $50 \%$ porosity) to silicates core volume ratio ranging from 2 to 10 .

ice mixtures on astronomical timescales are often lacking even though they provide valuable information and allow the rejection of some proposed fits, especially for the CO red component.

For example in Kerr et al. (1993), the fit of the red component with an $\mathrm{H}_{2} \mathrm{O}: \mathrm{CO}=20: 1$ ice mixture at $50 \mathrm{~K}$ spectrum is not compatible with the $\mathrm{H}_{2} \mathrm{O} / \mathrm{CO}_{\text {polar }} \approx 12$ column density ratio deduced from the integrated absorbances of the features.

Another example is the fit of L1489 IRS in Chiar et al. (1998) using an $\mathrm{H}_{2} \mathrm{O}: \mathrm{CO}=4: 1$ at $100 \mathrm{~K}$. The $\mathrm{CO} / \mathrm{H}_{2} \mathrm{O}$ ratio indicated cannot exceed about $10 \%$ immediately after reaching this temperature, as the $\mathrm{CO}$ in excess has sublimated from the ice matrix and the $4: 1$ ratio is valid only at $10 \mathrm{~K}$ (Schmitt et al. 1989). In addition, the crystallisation timescale of $\mathrm{H}_{2} \mathrm{O}$ ice is of about 500 years at this temperature (Schmitt 1992), with the consequence that all the CO is ejected from the water ice matrix. Unless there is a mechanism to counterbalance this phenomenon, such a mixture can only exist on laboratory (days) timescales.

In general, to fit the $2136 \mathrm{~cm}^{-1}$ (red) component, the authors need to use a laboratory spectra of an $\mathrm{H}_{2} \mathrm{O} / \mathrm{CO}$ mixture above about $30-35 \mathrm{~K}$ if the starting mixture deposited was "strong" (i.e. with $\mathrm{H}_{2} \mathrm{O} / \mathrm{CO} \gtrsim 0.1$ ), or "weak" mixtures (i.e. with $\mathrm{H}_{2} \mathrm{O} / \mathrm{CO} \lesssim 0.1$ ) at lower temperatures. The temperature annealing for strong mixtures above $30-35 \mathrm{~K}$ is required to satisfy both (i) the shift in frequency with increasing temperature to lower wavenumbers to match the interstellar feature and (ii) to get rid of an accompanying band at $\sim 2152 \mathrm{~cm}^{-1}$ observed in laboratory spectra of "strong" mixtures that disappear around this temperature and that is lacking in astronomical spectra (e.g. Pontoppidan et al. 2003). Even taking into consideration many deposition parameters and ice structures such as presented by Fraser et al. (2005), whatever the deposition conditions and ice porosity, the $2136 \mathrm{~cm}^{-1}$ band has never been observed at $T$ lower than $\sim 30 \mathrm{~K}$ without the adjacent band at $2152 \mathrm{~cm}^{-1}$. Unless an hypothetical astronomical molecule (yet to be astronomically constrained) will selectively block the formation of this $\mathrm{CO}$ binding site, the temperature constraint on the red component is valid for strong mixtures, i.e., the polar CO feature should be at temperatures above 30-35 K. The peak position located at $\approx 2136.5 \mathrm{~cm}^{-1}$ implies that the $\mathrm{H}_{2} \mathrm{O} / \mathrm{CO}$ mixture must be weak $(\lesssim 0.085$, Schmitt et al. 1989) if a low temperature spectrum is to be used.

These facts all put a severe constraint on the maximum amount of $\mathrm{CO}$ possibly embedded in $\mathrm{H}_{2} \mathrm{O}$, merely that the ratio $\mathrm{CO}_{\text {polar }} / \mathrm{H}_{2} \mathrm{O} \lesssim 0.08-0.1$ (Schmitt 1992). This constraint becomes higher if other species are trapped, as they will compete with each other for the space available in the water ice matrix (e.g. Bar-Nun \& Owen 1998), unless other strong intermolecular interactions are created by their presence.

In the case of $\mathrm{H}_{2} \mathrm{O} / \mathrm{CO}$ mixed ice subjected to $\mathrm{UV} /$ ion irradiation or when $\mathrm{CO}$ is produced in ice mixture matrices (e.g. Sandford et al. 1988; Palumbo \& Strazzulla 1993; Mennella et al. 2004; Palumbo 2005), the appearance of the $2152 \mathrm{~cm}^{-1}$ band at low temperature, accompanying the $2136 \mathrm{~cm}^{-1}$ one, is a more complex issue depending on numerous parameters. The newly formed species strongly alter the previous $\mathrm{CO}: \mathrm{H}_{2} \mathrm{O}$ interactions, and the energy input of a few $\mathrm{eV} /$ molecule is rather high compared to the bond energy of water ice molecules $(\sim 0.5 \mathrm{eV}$, see Palumbo \& Strazzulla 1993 and references therein), also favouring non reactive matrix reorganisation and partial $\mathrm{CO}$ migration from interstitial $\mathrm{H}_{2} \mathrm{O}$ sites. The notion of low temperature is therefore difficult to apply in this case after the molecules have undergone excited electronic state transitions following ion or UV irradiation.

Nevertheless, some objects display a red to central component integrated absorption that makes their profiles particular and depart strongly from the pure central component (e.g. L1489 IRS, Reipurth 50). We show in the following that a moderate increase of the upper size of the MRN distribution must be considered as an alternative/complementary explanation for this red component. These growth effects have been ignored in the majority of the previous analyses of the $\mathrm{CO}$ feature even though they represent an important parameter in the 
decomposition of some profiles, in addition to the intermolecular interaction effects.

\subsection{Modeling astrophysical behaviour}

\subsubsection{Reipurth 50}

We have modeled the $\mathrm{CO}$ absorption profile observed along the line of sight of Reipurth 50 with an MRN (Mathis et al. 1977) power law grain size distribution of ellipsoids with a quadratic weighting scheme as described previously. The minimum size of the grains in the distribution was set to $0.005 \mu \mathrm{m}$, and the upper size was varied to obtain a reasonable fit for the red component of the spectrum. The adopted dielectric function was built for a well mixed composite material as presented in Fig. 3, from the known dielectric functions of the individual materials that enter into its composition. For Reipurth 50, we built a composite material made of $50 \%$ silicates and $50 \%$ ice by volume. For the ice, the best mixture is $\left(\mathrm{CO}: \mathrm{CO}_{2}=100: 26\right)$, whose optical constants are taken from Ehrenfreund et al. 1996. The admixture of a few percent of $\mathrm{CO}_{2}$ in the $\mathrm{CO}$ matrix allows a better fit of the blue side of the absorption profile, as suggested by Boogert et al. (2002). The complex dielectric function is calculated using the Bruggeman rule, but the Looyenga rule gives a similar result, as shown in Appendix D, where we also detail other possible effective medium theories (EMTs).

The best fit, obtained for an upper grain size of $2 \mu \mathrm{m}$, is displayed in Fig. 11. To make a full comparison, an additional Gaussian absorption at $4.62 \mu \mathrm{m}$ is added, corresponding to the absorption of the cyanate ion $\mathrm{OCN}^{-}$(see e.g. van Broekhuizen et al. 2004, and references therein). Apart from the remaining small substructure differences at 4.66 and $4.69 \mu \mathrm{m}$, the width and red asymmetry can be explained by this size increase.

\subsubsection{CRBR $2422.8-3423$}

The profile of CRBR 2422.8 - 3423 gives more direct evidence of grain growth. The $\mathrm{CO}$ profile toward this source presents a $\mathrm{P}$ Cygni profile, with the blue part above the expected classical continuum rise. This is shown in Fig. 1 of Thi et al. (2002) or Fig. 12. The spectrum was modeled with an MRN (Mathis et al. 1977) power law grain size distribution of ellipsoids with a quadratic weighting scheme and dielectric function as for Reipurth 50, except that here we used a pure $\mathrm{CO}$ ice. The minimum size of the grains in the distribution was set to $0.005 \mu \mathrm{m}$, the maximum to $0.85 \mu \mathrm{m}$. The moderate grain sizes increase with respect to the diffuse ISM values, producing a red wing asymmetry explains this peculiar profile. The same extinction spectrum is shown with an optical depth of 0.5 instead of the 5 required to fit CRBR 2422.8-3423. This is more seen along many lines of sight, with exceptionally high optical depth, accentuating the contrast that reveals this behaviour.

In Pontoppidan et al. (2005), the comparison of the observed ${ }^{13} \mathrm{CO}$ profile with laboratory data, although affected by strong telluric lines, suggests that the broadening of this line could be slightly higher than expected with pure $\mathrm{CO}$, and a mixture with $10-20 \% \mathrm{CO}_{2}$ may fit better. The effect of adding

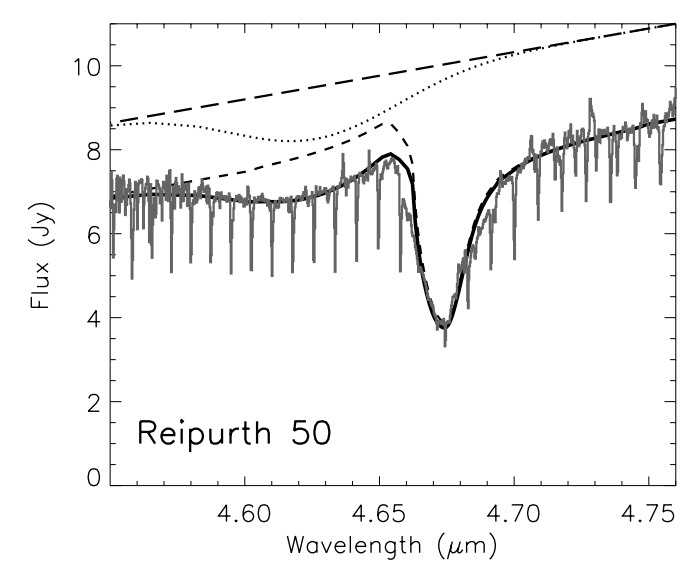

Fig. 11. CO ice band modeling of Reipurth 50. The source spectrum is displayed with the grey line; for a complete description of the observation, see Pontoppidan et al. (2003). The long dashed line show the local adopted continuum. The short dashed line is the modeled CO line profile using ellipsoids composed of equal parts of silicates and ices, with a quadratic weighting scheme. See the text for a more detailed explanation. The difference between the continuum and the extinction at e.g. $4.55 \mu \mathrm{m}$ is due to the presence of the refractive silicates. The full line fitted model includes an Gaussian-like absorption added to take the presence of the cyanide anion $\mathrm{OCN}^{-}$contribution into account.

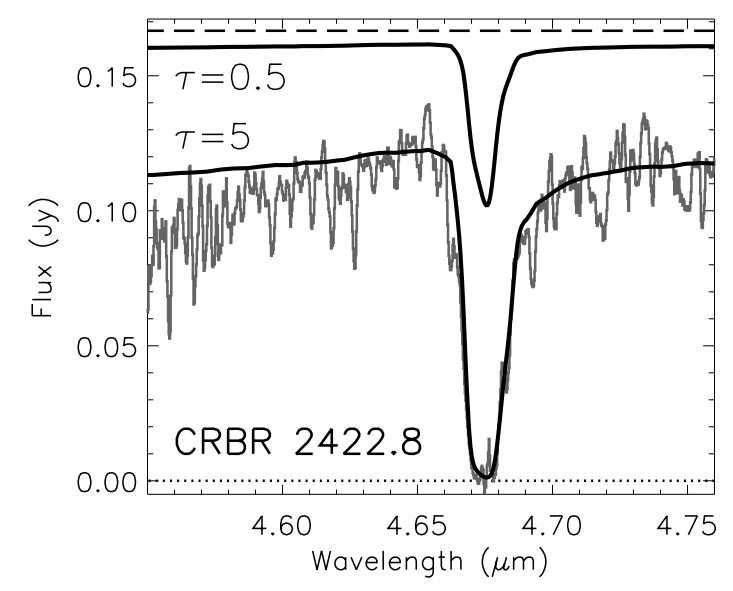

Fig. 12. CO ice band modeling of CRBR 2422.8 - 3423. The source spectrum is displayed with the grey line. For a complete description of the observation, see Thi et al. (2002). The long dashed line show the local adopted continuum. The short dashed line is the modeled CO line profile using ellipsoids composed of equal parts of silicates and ices, with a quadratic weighting scheme. See the text for a more detailed explanation. The difference between the continuum and the extinction at e.g. $4.55 \mu \mathrm{m}$ is due to the presence of the refractive silicates.

a few percent of $\mathrm{CO}_{2}$ mixed with $\mathrm{CO}$ would be the same as for the above fit of Reipurth 50 (see Fig. 11), i.e. allowing a slight extension of the extinction to the blue side $(\sim 4.66 \mu \mathrm{m})$ of the main $\mathrm{CO}$ band. Clearly a CRBR spectrum with a much higher signal to noise ratio is necessary to explore this further.

In the fits presented in this section, the aim is not to provide an exact determination of the $\mathrm{CO} / \mathrm{CO}_{2} /$ silicates proportions contributing to the observed bands, but rather to demonstrate that grain growth to moderate sizes (a few microns) by graingrain collision/coagulation is an alternative explanation for the 
appearance of a strong red wing in some of the observed $\mathrm{CO}$ ice profiles.

\section{Conclusion}

The volume attenuation coefficient of spherical and ellipsoidal silicates grains coated or mixed with an ice mantle made of pure or CO-dominated ice have been modeled to simulate the $\mathrm{CO}$ ice absorption profiles observed in dense media (clouds, disks) along astronomical lines of sight. The modeling was not limited to the classically used analytical formula in the Rayleigh limit but extended to arbitrary ellipsoidal shapes with size parameters up to 10 .

This modeling was applied to fit the astronomical spectra of two sources with peculiar CO profiles. It was shown that an increase of the grain size can explain the overall structure of the observed $\mathrm{CO}$ profile without the need to invoke specific mixtures or intermolecular interaction within ice mantles. This behaviour should be taken into account when one wants to extract more than an estimate of ice column densities. It does not however prevent us from also taking the intermolecular interactions into account when they are present and especially when they dominate the ice profiles of given modes (e.g. the $\mathrm{CO}_{2}$ bending mode toward W33 A or RAFGL 7009; Klotz et al. 2004; Dartois et al. 1999; Ehrenfreund et al. 1999).

The small grains, with respect to the size parameter at the observed wavelength, dominate the absorption centre of line profiles, whereas the intermediate grains will contribute to asymmetries in the wing. The behaviour of the volume polarisation term $\epsilon^{\prime}$ of the dielectric function first rising with frequency and then decreasing implies that, in the general case, the effects of scattering affect more efficiently the red part of the profile. Observing an absorption asymmetry that appears systematically in the red is therefore characteristic of light scattering in an absorption band for a grain with a size parameter around unity. At low optical depth, the continuum extraction generally assumes that there is no scattering effects and therefore often withdraw the blue wing shape by letting the continuum pass over it.

The pure ice grain models, adequate for the smallest grains, are not applicable on the overall typically assumed MRN interstellar grain size distribution. Grain growth to micron sizes contributes to the observed large red component in the $\mathrm{CO}$ absorption profiles toward many lines of sight, and traces the onset of scattering affecting the line profiles. Intermolecular interactions and grain growth are overlapping parameters that must be both taken into account to explain the detailed behaviour of infrared ice feature profiles in the dense regions where they are encountered and observed.

Acknowledgements. The author would like to thank W.-F. Thi, G. M. Muñoz Caro and S. Aparicio for fruitful discussions.

\section{References}

Adamson, A. J., Whittet, D. C. B., Chrysostomou, A., et al. 1999, ApJ, 512,224

Bar-Nun, A., \& Owen, T. 1998, Solar System Ices, ASSL, 227, 353
Baratta, G. A., \& Palumbo, M. E. 1998, Opt. Soc. Am. J. A, 15, 3076 Bhandari, R. 1985, Appl. Opt., 24, 1960

Boogert, A. C. A., Hogerheijde, M. R., \& Blake, G. A. 2002, ApJ, 568,761

Bohren, C. F., \& Huffman, D. R. 1983 (New York: Wiley), 1983

Bowey, J. E., Rawlings, M. G., \& Adamson, A. J. 2004, MNRAS, 348, L13

Bruggeman, D. A. G. 1925, Ann. Phys., 24, 636

Chang, H., Richardson, H. H., \& Ewing, G. E. 1988, J. Chem. Phys., 89,7561

Chiar, J. E., Gerakines, P. A., Whittet, D. C. B., et al. 1998, ApJ, 498, 716

Chiar, J. E., Adamson, A. J., Kerr, T. H., \& Whittet, D. C. B. 1995, ApJ, 455, 234

Dartois, E. 2005, Space Sci. Rev., accepted

Dartois, E., Cox, P., Roelfsema, P. R., et al. 1998, A\&A, 338, L21

Dartois, E., Demyk, K., d'Hendecourt, L., \& Ehrenfreund, P. 1999, A\&A, 351, 1066

Draine, B. T., \& Flatau, P. J. 2003, User Guide to the Discrete Dipole Approximation Code DDSCAT.6.0, http://arxiv.org/abs/astro-ph/0300969

Draine, B. T., \& Lee, H. M. 1984, ApJ, 285, 89

Ehrenfreund, P., Kerkhof, O., Schutte, W. A., et al. 1999, A\&A, 350, 240

Ehrenfreund, P., Boogert, A. C. A., Gerakines, P. A., et al. 1996, A\&A, 315, L341

Eiroa, C., \& Hodapp, K.-W. 1989, A\&A, 210, 345

Elsila, J., Allamandola, L. J., \& Sandford, S. A. 1997, ApJ, 479, 818

Evans, A. 1993, The Dusty Universe, Ellis Horwood, 89

Fabian, D., Henning, T., Jäger, C., Mutschke, H., Dorschner, J., \& Wehrhan, O. 2001, A\&A, 378, 228

McFadzean, A. D., Whittet, D. C. B., Bode, M. F., Adamson, A. J., \& Longmore, A. J. 1989, MNRAS, 241, 873

Fraser, H. J., Bisschop, S. E., Pontoppidan, K. M., Tielens, A. G. G. M., \& van Dishoeck, E. F. 2005, MNRAS, 356, 1283

Geballe, T. R. 1986, A\&A, 162, 248

Gerakines, P. A., Schutte, W. A., Greenberg, J. M., \& van Dishoeck, E. F. 1995 , A\&A, 296, 810

Gibb, E. L., Whittet, D. C. B., Boogert, A. C. A., \& Tielens, A. G. G. M. 2004, ApJS, 151, 35

Gillett, F. C., \& Forrest, W. J. 1973, ApJ, 179, 483

Dhendecourt, L. B., \& Allamandola, L. J. 1986, A\&AS, 64, 453

Kai, L., \& Massoli, P. 1994, Appl. Opt., 33, 501

Kerr, T. H., Adamson, A. J., \& Whittet, D. C. B. 1993, MNRAS, 262, 1047

Kerr, T. H., Adamson, A. J., \& Whittet, D. C. B. 1991, MNRAS, 251, $60 \mathrm{P}$

Klotz, A., Ward, T., \& Dartois, E. 2004, A\&A, 416, 801

Lacy, J. H., Baas, F., Allamandola, L. J., et al. 1984, ApJ, 276, 533

Lichtenecker, K. 1926, Phys. Z., 27, 115

Looyenga, H. 1965, Phys., 31, 401

Maxwell-Garnett J. C. 1904 Philos. Trans. R. Soc. London, 385

Mennella, V., Palumbo, M. E., \& Baratta, G. A. 2004, ApJ, 615, 1073

Merrill, K. M., Russell, R. W., \& Soifer, B. T. 1976, ApJ, 207, 763

Mathis, J. S., Rumpl, W., \& Nordsieck, K. H. 1977, ApJ, 217, 425

Murakawa, K., Tamura, M., \& Nagata, T. 2000, ApJS, 128, 603

Palumbo, M. E. 2005, J. Phys. Conf. Ser., 6, 211

Palumbo, M. E., \& Strazzulla, G. 1993, A\&A, 269, 568

Pontoppidan, K. M., Dullemond, C. P., van Dishoeck, E. F., et al. 2005, ApJ, 622, 463

Pontoppidan, K. M., Fraser, H. J., Dartois, E., et al. 2003, A\&A, 408, 981

Reddy, R. R. Rama Gopal, K., Nazeer Ahammed, Y., et al. 2004, Solid State Ionics, in Press 
Rieke, G. H., \& Lebofsky, M. J. 1985, ApJ, 288, 618

Sandford, S. A., Allamandola, L. J., Tielens, A. G. G. M., \& Valero, G. J. 1988, ApJ, 329, 498

Scarrott, S. M., Rolph, C. D., \& Semple, D. P. 1990, Galactic and Intergalactic Magnetic Fields, IAU Symp., 140, 245

Schmitt, B., Greenberg, J. M., \& Grim, R. J. A. 1989, ApJ, 340, L33

Schmitt, B. 1992, Interrelations Between Physics and Dynamics for Minor Bodies in the Solar System, 265

Smith, R. G., Sellgren, K., \& Tokunaga, A. T. 1989, ApJ, 344, 413

Swanepoel, R. 1983, J. Phys. E Scientific Instruments, 16, 1214

Tanaka, M., Nagata, T., Sato, S., \& Yamamoto, T. 1994, ApJ, 430, 779

Teixeira, T. C., Emerson, J. P., \& Palumbo, M. E. 1998, A\&A, 330, 711

Thi, W. F., Pontoppidan, K. M., van Dishoeck, E. F., Dartois, E., \& d'Hendecourt, L. 2002, A\&A, 394, L27

Tielens, A. G. G. M., Tokunaga, A. T., Geballe, T. R., \& Baas, F. 1991, ApJ, 381, 181
Toon, O. B., \& Ackerman, T. P. 1981, Appl. Opt., 20, 3657

Trotta, F. 1996, Ph.D. Thesis, Université Joseph Fourier, Grenoble van Broekhuizen, F. A., Keane, J. V., \& Schutte, W. A. 2004, A\&A, 415,425

van Dishoeck, E. F. 2004, ARA\&A, 42, 119

Willner, S. P., Gillett, F. C., Herter, T. L., et al. 1982, ApJ, 253, 174

Whittet, D. C. B., Adamson, A. J., Duley, W. W., Geballe, T. R., \& McFadzean, A. D. 1989, MNRAS, 241, 707

Whittet, D. C. B., McFadzean, A. D., \& Longmore, A. J. 1985, MNRAS, 216, 45

Whittet, D. C. B., Bode, M. F., Baines, D. W. T., Longmore, A. J., \& Evans, A. 1983, Nature, 303, 218

Wu, Z. S., Guo, L., Ren, K. F., Gouesbet, G., \& Gréhan, G. 1997, Appl. Opt., 36, 5188

Yang, W 2003, Appl. Opt., 42, 1710

Zubko, V. G., Mennella, V., Colangeli, L., \& Bussoletti, E. 1996, MNRAS, 282, 1321 


\section{Online Material}




\section{Appendix A: Rayleigh regime cross sections}

\section{A.1. Homogeneous spheres}

The Rayleigh cross sections for spheres can be found in Bohren \& Huffman 1983 (Sect. 5.2), and are given by:

$$
\begin{gathered}
C_{\mathrm{ext}}=\mathrm{k} \operatorname{Im}(\alpha)=\pi \mathrm{a}^{2} 4 x \operatorname{Im}\left(\frac{\epsilon_{1}-\epsilon_{\mathrm{m}}}{\epsilon_{1}+2 \epsilon_{\mathrm{m}}}\right) \\
C_{\mathrm{sca}}=\frac{\mathrm{k}^{4}}{6 \pi}|\alpha|^{2}=\pi \mathrm{a}^{2} \frac{8}{3} x^{4}\left|\frac{\epsilon_{1}-\epsilon_{\mathrm{m}}}{\epsilon_{1}+2 \epsilon_{\mathrm{m}}}\right|^{2}
\end{gathered}
$$

where $C_{\text {ext }}, C_{\text {sca }}, C_{\text {abs }}$ are the extinction, scattering and absorption cross sections $\left(\left[\mathrm{cm}^{2}\right]\right)$, a is the sphere radius, $\epsilon_{1}$ and $\epsilon_{m}$ are the complex dielectric functions of the material constituting the sphere and of the outer medium, respectively, and $x=k a=\frac{2 \pi a}{\lambda}$ is the size parameter, where $\lambda$ is the wavelength considered. In the Rayleigh regime, by definition, $x \ll 1$, then $C_{\text {sca }} \ll C_{\text {abs }}$ and therefore $C_{\mathrm{ext}} \approx C_{\mathrm{abs}}$. Using $v=\frac{4}{3} \pi a^{3}$ one obtains:

$$
C_{\mathrm{ext}}^{\mathrm{HS}} \approx C_{\mathrm{abs}}^{\mathrm{HS}}=3 k v \operatorname{Im}\left(\frac{\epsilon_{1}-\epsilon_{m}}{\epsilon_{1}+2 \epsilon_{m}}\right) .
$$

\section{A.2. Coated spheres}

The Rayleigh cross sections for coated spheres can be found in Bohren \& Huffman 1983 (Sect. 5.4), and are given by:

$C_{\mathrm{abs}}=\mathrm{k} \operatorname{Im}(\alpha)$

$\frac{\alpha}{v}=3 \frac{\left(\epsilon_{2}-\epsilon_{m}\right)\left(\epsilon_{1}+2 \epsilon_{2}\right)+f\left(\epsilon_{1}-\epsilon_{2}\right)\left(\epsilon_{m}+2 \epsilon_{2}\right)}{\left(\epsilon_{2}+2 \epsilon_{m}\right)\left(\epsilon_{1}+2 \epsilon_{2}\right)+f\left(2 \epsilon_{2}-2 \epsilon_{m}\right)\left(\epsilon_{1}-\epsilon_{2}\right)}$

$v=\frac{4}{3} \pi a_{2}^{3} \quad f=\left(\frac{a_{1}}{a_{2}}\right)^{3}$

where $a_{1}$ and $a_{2}$ are the sphere radius corresponding to the core and the mantle, $f$ is the inner to total volume ratio, $\epsilon_{1}, \epsilon_{2}$ and $\epsilon_{m}$ are the complex dielectric functions of the core, mantle and outer medium, respectively and $k=\frac{2 \pi}{\lambda}, \lambda$ is the wavelength considered. $f$ is related to the mantle to core volume ratio by $f_{\text {vol }}=\frac{V(\text { ice })}{V(\text { sil })}=\frac{1}{f}-1$.

$C_{\mathrm{abs}}^{\mathrm{CS}}=3 k v \operatorname{Im}\left(\frac{\left(\epsilon_{2}-\epsilon_{m}\right)\left(\epsilon_{1}+2 \epsilon_{2}\right)+f\left(\epsilon_{1}-\epsilon_{2}\right)\left(\epsilon_{m}+2 \epsilon_{2}\right)}{\left(\epsilon_{2}+2 \epsilon_{m}\right)\left(\epsilon_{1}+2 \epsilon_{2}\right)+f\left(2 \epsilon_{2}-2 \epsilon_{m}\right)\left(\epsilon_{1}-\epsilon_{2}\right)}\right)$.

\section{A.3. Homogeneous identical ellipsoids}

The average absorption cross section of randomly oriented identical homogeneous ellipsoids particles in the Rayleigh regime (e.g. Bohren \& Huffman 1983, Sect. 5.5.1) is given by:

$\left\langle C_{\mathrm{abs}}^{\mathrm{abc}}\right\rangle=k \operatorname{Im}\left(\frac{\alpha_{1}+\alpha_{2}+\alpha_{3}}{3}\right)$

with:

$$
\begin{aligned}
& \frac{\alpha_{i}}{v}=\frac{1}{\beta+L_{i}} \quad i=1,2,3 \quad \beta=\frac{1}{\epsilon_{\mathrm{rel}}-1} \quad \epsilon_{\mathrm{rel}}=\frac{\epsilon_{1}}{\epsilon_{\mathrm{m}}} \\
& L_{1}=\frac{a b c}{2} \int_{0}^{\infty} \frac{\mathrm{d} q}{\left(a^{2}+q\right) f(q)} ; L_{2}=\frac{a b c}{2} \int_{0}^{\infty} \frac{\mathrm{d} q}{\left(b^{2}+q\right) f(q)}
\end{aligned}
$$

$$
\begin{aligned}
& L_{3}=\frac{a b c}{2} \int_{0}^{\infty} \frac{\mathrm{d} q}{\left(c^{2}+q\right) f(q)} ; f(q)=\sqrt{\left(a^{2}+q\right)\left(b^{2}+q\right)\left(c^{2}+q\right)} \\
& L_{1}+L_{2}+L_{3}=1
\end{aligned}
$$

where $a, b, c$ are three principal axes of the considered ellipsoid, $v$ the volume of the ellipsoid, $\epsilon_{1}$ and $\epsilon_{m}$ are the complex dielectric functions of the ellipsoid and outer medium, respectively. $k=\frac{2 \pi}{\lambda}$, where $\lambda$ is the wavelength considered. $L_{1}, L_{2}$ and $L_{3}$ are the geometrical factors associated with each principal axis.

\section{A.4. Continuous distribution of homogeneous ellipsoids}

To take into account a distribution of all possible ellipsoids shapes, the randomly oriented identical ellipsoid cross section presented above has to be averaged over the sets of geometrical factors $L_{1}, L_{2}$ and $L_{3}$ that define a unique ellipsoid shape, weighted by the probability $P\left(L_{1}, L_{2}, L_{3}\right)$ of finding such a given shape. Using the dependence between geometrical factors $\left(L_{1}+L_{2}+L_{3}=1\right)$, this average, the Weighted Continuous Distribution of Ellipsoids (WCDE) over the shape distribution and orientations reduces to:

$\left\langle\left\langle C_{\mathrm{abs}}^{\mathrm{WCDE}}\right\rangle\right\rangle=\int_{0}^{1} \mathrm{~d} L_{1} \int_{0}^{1-L_{1}}\left\langle C_{\mathrm{abs}}\right\rangle P\left(L_{1}, L_{2}\right) \mathrm{d} L_{2}$

with:

$\int_{0}^{1} \mathrm{~d} L_{1} \int_{0}^{1-L_{1}} P\left(L_{1}, L_{2}\right) \mathrm{d} L_{2}=1$.

\section{A.4.1. Uniform weighting}

For uniform shape probability $P\left(L_{1}, L_{2}\right)=2$ therefore:

$\left\langle\left\langle C_{\mathrm{abs}}^{\mathrm{UCDE}}\right\rangle\right\rangle=k v \operatorname{Im}\left(\frac{2 \epsilon_{\mathrm{rel}}}{\epsilon_{\mathrm{rel}}-1} \ln \epsilon_{\mathrm{rel}}\right)$

where $\epsilon_{\mathrm{rel}}, k$ and $v$ have the same meanings as defined previously. This weighting is in fact physically unjustified, as it implies an infinitely long and thin cylindric-like ellipsoid has the same probability to occur than a sphere or close to sphere shape one. In the real world, collisions and or accretion will tend either to eliminate the extreme ellipsoid shape or favor the spheroidal one. In other words, the classical uniform CDE model should not be used as the "best case" unless it has been demonstrated by physical means that the extreme shapes taken explicitly into account in such a distribution indeed contribute significantly to the shape distribution.

\section{A.4.2. Quadratic weighting}

Perhaps a more adapted and realistic weighting function is $P\left(L_{1}, L_{2}, L_{3}\right) \propto L_{1} L_{2} L_{3}=L_{1} L_{2}\left(1-L_{1}-L_{2}\right)$, leading to the quadratic weighting continuous distribution of ellipsoids (QCDE). With such a weighting, due to the interdependence of geometrical factors, if one axis of the ellipsoid is much away from the others, i.e. when the ellipsoid tend to be like an infinite rod or a plane, the probability drops. Extreme shapes 
become therefore less probable. Such a weighting scheme has been used by e.g. Fabian et al. (2001) and is given by:

$\left\langle\left\langle C_{\mathrm{abs}}^{\mathrm{QCDE}}\right\rangle\right\rangle=$

$C \frac{k v}{3} \operatorname{Im}\left[\sum_{\mathrm{i}=1}^{3} \int_{0}^{1} \mathrm{~d} L_{1} \int_{0}^{1-L_{1}} \frac{L_{1} L_{2}\left(1-L_{1}-L_{2}\right)}{\beta+L_{\mathrm{i}}} \mathrm{d} L_{2}\right]$

$C$ being a constant to satisfy the normalization of the shape probability function (here $C=20$ ). Rearranging the equations and using the symmetries of the function one obtains:

$\left\langle\left\langle C_{\mathrm{abs}}^{\mathrm{QCDE}}\right\rangle\right\rangle=C k v \operatorname{Im}\left[\int_{0}^{1} \frac{L_{1}\left(1-L_{1}\right)^{3}}{\beta+L_{1}} \mathrm{~d} L_{1}\right]$

$\left\langle\left\langle C_{\mathrm{abs}}^{\mathrm{QCDE}}\right\rangle\right\rangle=C k v \operatorname{Im}\left[\int_{0}^{1} \frac{-L_{1}^{4}+3 L_{1}^{3}-3 L_{1}^{2}+L_{1}}{\beta+L_{1}} \mathrm{~d} L_{1}\right]$.

The integral can be reorganised by decomposing the fraction into a sum of polynomials as follows:

$$
\begin{array}{r}
\int_{0}^{1} \frac{-L_{1}^{4}+3 L_{1}^{3}-3 L_{1}^{2}+L_{1}}{\beta+L_{1}} \mathrm{~d} L_{1}=\int_{0}^{1}\left[-L_{1}^{3}+(3+\beta) L_{1}^{2}\right. \\
\left.-\left(\beta^{2}+3 \beta+3\right) L_{1}+(\beta+1)^{3}-\frac{\beta(\beta+1)^{3}}{\beta+L_{1}}\right] \mathrm{d} L_{1}
\end{array}
$$

and by making the variable change $\gamma=\beta+L_{1}, \mathrm{~d} \gamma=\mathrm{d} L_{1}$ and integral limits $0 \rightarrow \beta$ and $1 \rightarrow \beta+1$, it leads to the result:

$$
\left\langle\left\langle C_{\mathrm{abs}}^{\mathrm{QCDE}}\right\rangle\right\rangle=20 k v \operatorname{Im}\left[-\beta \epsilon_{\mathrm{rel}}^{3} \ln \left(\epsilon_{\mathrm{rel}}\right)+\frac{1}{4}+\frac{11}{6} \beta+\frac{5}{2} \beta^{2}+\beta^{3}\right]
$$

with $\beta=\frac{1}{\epsilon_{\mathrm{rel}}-1}$.

\section{A.5. Coated identical ellipsoids}

The average absorption cross section of randomly oriented identical coated ellipsoids particles in the Rayleigh regime is given by:

$$
\left\langle C_{\mathrm{abs}}^{\mathrm{a}_{2} \mathrm{~b}_{2} \mathrm{c}_{2}}\right\rangle=k \operatorname{Im}\left(\frac{\alpha_{1}+\alpha_{2}+\alpha_{3}}{3}\right)
$$

with

$\frac{\alpha_{i}}{\mathrm{~V}}=$

$\frac{\left(\epsilon_{2}-\epsilon_{\mathrm{m}}\right)\left[\epsilon_{2}+\left(\epsilon_{1}-\epsilon_{2}\right)\left(L_{\mathrm{i}}^{\mathrm{int}}-f L_{\mathrm{i}}^{\mathrm{ext}}\right)\right]+f \epsilon_{2}\left(\epsilon_{1}-\epsilon_{2}\right)}{\left[\epsilon_{2}+\left(\epsilon_{1}-\epsilon_{2}\right)\left(L_{\mathrm{i}}^{\mathrm{int}}-f L_{\mathrm{i}}^{\mathrm{ext}}\right)\right]\left[\epsilon_{\mathrm{m}}+\left(\epsilon_{2}-\epsilon_{\mathrm{m}}\right) L_{\mathrm{i}}^{\mathrm{ext}}\right]+f L_{\mathrm{i}}^{\mathrm{ext}} \epsilon_{2}\left(\epsilon_{1}-\epsilon_{2}\right)}$

$i=1,2,3 \quad v=\frac{4}{3} \pi a_{2} b_{2} c_{2} \quad f=\frac{a_{1} b_{1} c_{1}}{a_{2} b_{2} c_{2}}$

where $a_{1}, b_{1}, c_{1}$ and $a_{2}, b_{2}, c_{2}$ define the inner (core) and outer (mantle) axes, respectively. The complex dielectric functions $\epsilon_{1}$ and $\epsilon_{2}$ are related to the core and mantle constituents. $v$ is the volume of the ellipsoid and $f$ the volume ratio of core to total volume. The various geometrical factors entering into the equations are defined as follow:

$L_{1}^{\mathrm{int}}=\frac{a_{1} b_{1} c_{1}}{2} \int_{0}^{\infty} \frac{\mathrm{d} q}{\left(a_{1}^{2}+q\right) f_{1}(q)}$
$L_{1}^{\mathrm{ext}}=\frac{a_{2} b_{2} c_{2}}{2} \int_{0}^{\infty} \frac{\mathrm{d} q}{\left(a_{2}^{2}+q\right) f_{2}(q)}$

$L_{2}^{\mathrm{int}}=\frac{a_{1} b_{1} c_{1}}{2} \int_{0}^{\infty} \frac{\mathrm{d} q}{\left(b_{1}^{2}+q\right) f_{1}(q)} ;$

$L_{2}^{\mathrm{ext}}=\frac{a_{2} b_{2} c_{2}}{2} \int_{0}^{\infty} \frac{\mathrm{d} q}{\left(b_{2}^{2}+q\right) f_{2}(q)}$

$L_{3}^{\mathrm{int}}=\frac{a_{1} b_{1} c_{1}}{2} \int_{0}^{\infty} \frac{\mathrm{d} q}{\left(c_{1}^{2}+q\right) f_{1}(q)}$

$L_{3}^{\mathrm{ext}}=\frac{a_{2} b_{2} c_{2}}{2} \int_{0}^{\infty} \frac{\mathrm{d} q}{\left(c_{2}^{2}+q\right) f_{2}(q)}$

$f_{1}(q)=\sqrt{\left(a_{1}^{2}+q\right)\left(b_{1}^{2}+q\right)\left(c_{1}^{2}+q\right)} \quad L_{1}^{\mathrm{int}}+L_{2}^{\mathrm{int}}+L_{3}^{\mathrm{int}}=1$

$f_{2}(q)=\sqrt{\left(a_{2}^{2}+q\right)\left(b_{2}^{2}+q\right)\left(c_{2}^{2}+q\right)} \quad L_{1}^{\mathrm{ext}}+L_{2}^{\mathrm{ext}}+L_{3}^{\mathrm{ext}}=1$.

An analytic expression to account for a distribution of coated ellipsoids shapes is often impossible to evaluate and the mean cross section is therefore estimated numerically.

\section{Appendix B: Adopted continuous distribution of coated ellipsoids}

The continuous distribution of ellipsoids used in the modeling presented in the article is not limited to the Rayleigh regime. For a given coated ellipsoid, defined by its inner core parameters (axes $a_{1}, b_{1}, c_{1}$, complex dielectric function $\epsilon_{1}$ ) and outer mantle (axes $a_{2}, b_{2}, c_{2}$, complex dielectric function $\epsilon_{2}$ ) and a size parameter $x=\frac{2 \pi\langle a\rangle}{\lambda}$, where $\langle a\rangle$ is the equivalent radius of a sphere of the same volume that the considered ellipsoid, we calculate the resulting orientation average of the cross section, $\left\langle C_{\text {ext }}\left(L_{1}, L_{2}\right)\right\rangle$, where $L_{1}, L_{2}, L_{3}=1-L_{1}-L_{2}$ are the geometrical factors already defined in Appendix A, and related to $a_{2}, b_{2}, c_{2}$. The size parameter value is defined strictly for a wavelength of $4.67 \mu \mathrm{m}$, as the calculations are performed at fixed grain radius for obvious physical reasons, over the $[4.6,4.75] \mu \mathrm{m}$ wavelength range .

To model the individual coated ellipsoid, we make use of the DDA program DDSCAT ${ }^{3}$ (Draine \& Flatau 2003).

Following the quadratic weighting prescription already presented for homogeneous ellipsoids we wish to calculate:

$\left\langle\left\langle C_{\mathrm{ext}}^{\mathrm{QCDE}}(\lambda)\right\rangle\right\rangle=\int_{0}^{1} \mathrm{~d} L_{1} \int_{0}^{1-L_{1}}\left\langle C_{\text {ext }}(\lambda)\right\rangle P\left(L_{1}, L_{2}\right) \mathrm{d} L_{2}$

where $P\left(L_{1}, L_{2}\right)=\gamma L_{1} L_{2} L_{3}=\gamma L_{1} L_{2}\left(1-L_{1}-L_{2}\right)$, and

$\frac{1}{\gamma}=\int_{0}^{1} \mathrm{~d} L_{1} \int_{0}^{1-L_{1}} L_{1} L_{2}\left(1-L_{1}-L_{2}\right) \mathrm{d} L_{2}$

Calculations are performed over all possible ellipsoids with relative non-degenerated integer aspect ratios of the three axes

\footnotetext{
3 A description of the program can be found at http://www.astro.princeton.edu/ draine
} 


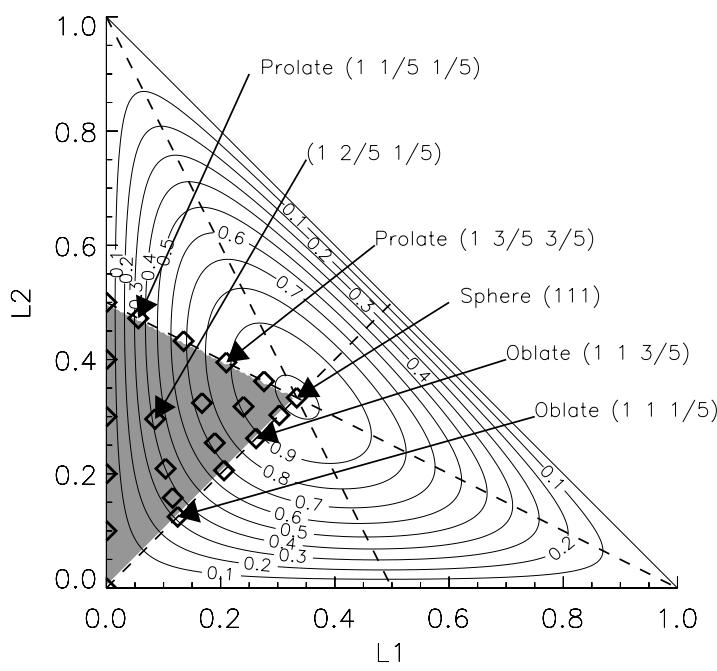

Fig. B.1. Shape probability function $P\left(L_{1}, L_{2}\right)$ for a quadratic weighting scheme as explained in the text, as a function of the two geometrical factors $L_{1}$ and $L_{2}$ ( $L_{3}$ is implicitly defined by $\left.L_{3}=1-L_{1}-L_{2}\right)$. Contours levels displayed correspond to $\{0.1,0.2,0.3,0.4,0.5,0.6,0.7,0.8,0.9,0.99\}$, the function displayed being normalised here to the sphere probability of 1 . The grey scale sextant is the probability domain of definition (see e.g. Bohren \& Huffman 1983, Sect. 12.2.4 for further explanation). The diamonds represent the locations of ellipsoid cross sections evaluated using the DDSCAT program, except for the ones on the $L_{1}=0$ axis, which are points (with $P\left(L_{1}, L_{2}\right)=0$ ) added for convenience to perform triangulation. Examples of specific ellipsoids are shown with arrows and their corresponding normalised axes ratios are explicitly given. The $L_{1}=1-2 L_{2}$ line delineates the prolate spheroid asymptotic limit whereas the $L_{1}=L_{2}$ one delineates the oblate spheroid one.

(with $a \geq b \geq c$ ) between 1 and 5 , having fixed the first axis to the highest integer ( 15 possibilities in total). The orientation average of the cross section for each ellipsoid, is performed over 16 angles equally sampling the relative orientation of individual ellipsoids with respect to the incoming plane wave. The locations of the calculated $\left\langle C_{\text {ext }}(\lambda)\right\rangle$ in the $\left\{L_{1}, L_{2}\right\}$ plane are displayed in Fig. B.1.

The resultant quadratic weighted continuous distribution of ellipsoids is obtained by weighting each orientations average calculated at a given wavelength $\left\langle C_{\text {ext }}(\lambda)\right\rangle$ of each ellipsoid shape by its shape probability $P\left(L_{1}, L_{2}\right)$ (displayed as contours in Fig. B.1). The function in the sextant of the $L_{1}, L_{2}$ plane (grey scale in Fig. B.1), which defines the domain of definition for the shape probability function $P\left(L_{1}, L_{2}\right)$, is therefore obtained by triangulation from the measured points, and integrated numerically. The operation is repeated for each wavelength at fixed equivalent total grain radius.

\section{Appendix C: Multi-layered sphere with arbitrary size parameter}

For the general multi-layered sphere case, we follow the excellent improved recursive algorithm of Yang (2003) that allows calculations of the scattering coefficients for a multilayered sphere with large size parameters without significant computational errors. The scattering coefficients are calculated according to:

$$
\begin{aligned}
& C_{\text {ext }}=\frac{2 \pi}{k^{2}} \sum_{n=1}^{N \text { stop }}(2 n+1) \operatorname{Re}\left\{a_{n}+b_{n}\right\} \\
& C_{\text {sca }}=\frac{2 \pi}{k^{2}} \sum_{n=1}^{N \text { stop }}(2 n+1)\left(\left|a_{n}\right|^{2}+\left|b_{n}\right|^{2}\right)
\end{aligned}
$$

$N$ stop defines the limit needed to describe the wave expansion series above, and is size parameter dependent as follows:

$$
\text { Nstop }=\begin{array}{lr}
x_{L}+4 x_{L}^{1 / 3}+1 & 0.02 \leq x_{L} \leq 8 \\
x_{L}+4.05 x_{L}^{1 / 3}+2 & 8 \leq x_{L} \leq 4200 \\
x_{L}+4 x_{L}^{1 / 3}+2 & 4200 \leq x_{L} \leq 20000
\end{array}
$$

$k=2 \pi / \lambda, \lambda$ being the wavelength, and:

$a_{n}=\frac{\left[H_{n}^{a}\left(m_{L} x_{L}\right) / m_{L}+n / x_{L}\right] \psi_{n}\left(x_{L}\right)-\psi_{n-1}\left(x_{L}\right)}{\left[H_{n}^{a}\left(m_{L} x_{L}\right) / m_{L}+n / x_{L}\right] \zeta_{n}\left(x_{L}\right)-\zeta_{n-1}\left(x_{L}\right)}$

$b_{n}=\frac{\left[m_{L} H_{n}^{a}\left(m_{L} x_{L}\right)+n / x_{L}\right] \psi_{n}\left(x_{L}\right)-\psi_{n-1}\left(x_{L}\right)}{\left[m_{L} H_{n}^{a}\left(m_{L} x_{L}\right)+n / x_{L}\right] \zeta_{n}\left(x_{L}\right)-\zeta_{n-1}\left(x_{L}\right)}$

with $x_{l}=k N r_{l}$ and $m_{l}=N_{l} / N=\left(n_{l}+i k_{l}\right), l=1, \ldots, L$ is the index referring to the $l$ st layer of radius $r_{l}$, the last layer being $\mathrm{L}$. $N$ is the refractive index of the external medium. $\psi_{n}(z)=z j_{n}(z)$ and $\zeta_{n}(z)=z\left[j_{n}(z)+i y_{n}(z)\right]$ are the Riccati-Bessel functions, where $j_{n}(z)$ and $y_{n}(z)$ are the spherical Bessel functions of first and second kind, respectively.

$$
\begin{aligned}
& H_{n}^{a}\left(m_{l} x_{l}\right)=\frac{G_{2} D_{n}^{(1)}\left(m_{l} x_{l}\right)-G_{1} Q_{n}^{(l)} D_{n}^{(3)}\left(m_{l} x_{l}\right)}{G_{2}-G_{1} Q_{n}^{(l)}} \\
& H_{n}^{b}\left(m_{l} x_{l}\right)=\frac{G_{4} D_{n}^{(1)}\left(m_{l} x_{l}\right)-G_{3} Q_{n}^{(l)} D_{n}^{(3)}\left(m_{l} x_{l}\right)}{G_{4}-G_{3} Q_{n}^{(l)}} \\
& G 1=m_{l} H_{n}^{a}\left(m_{l-1} x_{l-1}\right)-m_{l-1} D_{n}^{(1)}\left(m_{l} x_{l-1}\right) \\
& G 2=m_{l} H_{n}^{a}\left(m_{l-1} x_{l-1}\right)-m_{l-1} D_{n}^{(3)}\left(m_{l} x_{l-1}\right) \\
& G 3=m_{l-1} H_{n}^{b}\left(m_{l-1} x_{l-1}\right)-m_{l} D_{n}^{(1)}\left(m_{l} x_{l-1}\right) \\
& G 4=m_{l-1} H_{n}^{b}\left(m_{l-1} x_{l-1}\right)-m_{l} D_{n}^{(3)}\left(m_{l} x_{l-1}\right) \\
& Q_{n}^{(l)}=\frac{\psi_{n}\left(m_{l} x_{l-1}\right)}{\zeta_{n}\left(m_{l} x_{l-1}\right)} / \frac{\psi_{n}\left(m_{l} x_{l}\right)}{\zeta_{n}\left(m_{l} x_{l}\right)}
\end{aligned}
$$

$D_{n}^{(1)}(z)=\psi_{n}^{\prime}(z) / \psi_{n}(z)$ is calculated by downward recurrence as follows:

$$
\begin{aligned}
& N \max =N \text { stop }+15 \\
& D_{N \max }^{(1)}(z)=0.0+0.0 \mathrm{i} \\
& D_{n-1}^{(1)}(z)=n / z-1 /\left(D_{n}^{(1)}(z)+n / z\right)
\end{aligned}
$$

$D_{n}^{(3)}(z)=\zeta_{n}^{\prime}(z) / \zeta_{n}(z)$ is calculated by upward recurrence as follows:

$$
\begin{aligned}
& D_{0}^{(3)}(z)=0.0+1.0 \mathrm{i} \\
& \psi_{0}(z) \zeta_{0}(z)=-\mathrm{i} \exp (\mathrm{i} z) \sin (z) \\
& \psi_{n}(z) \zeta_{n}(z)=\psi_{n-1}(z) \zeta_{n-1}(z)\left(n / z-D_{n-1}^{(1)}(z)\right)\left(n / z-D_{n-1}^{(3)}(z)\right) \\
& D_{n}^{(3)}(z)=D_{n}^{(1)}(z)+\mathrm{i} /\left[\psi_{n}(z) \zeta_{n}(z)\right]
\end{aligned}
$$


E. Dartois: Spectroscopic evidence of grain ice mantle growth in YSOs. I., Online Material p 5

$Q_{n}^{(l)}$ is calculated by upward recurrence as follows:

$Q_{0}^{(1)}=\frac{\exp \left(-\mathrm{i} 2 a_{1}\right)-\exp \left(-2 b_{1}\right)}{\exp \left(-\mathrm{i} 2 a_{2}\right)-\exp \left(-2 b_{2}\right)} \exp \left[-2\left(b_{2}-b_{1}\right)\right]$

$z_{1}=m_{l} x_{l-1}=a_{1}+\mathrm{i} b_{1} \quad z_{2}=m_{l} x_{l}=a_{2}+\mathrm{i} b_{2}$

$Q_{n}^{(\mathrm{l})}=Q_{n-1}^{(\mathrm{l})}\left(\frac{z_{2}}{z_{1}}\right)^{2}\left(\frac{z_{2} D_{n}^{(1)}\left(z_{2}\right)+n}{z_{1} D_{n}^{(1)}\left(z_{1}\right)+n}\right)\left(\frac{n-z_{2} D_{n-1}^{(3)}\left(z_{2}\right)}{n-z_{1} D_{n-1}^{(3)}\left(z_{1}\right)}\right)$

and the classical Riccati-Bessel function are evaluated by the following upward recurrence:

$\zeta_{-1}(z)=\cos (z)+i \sin (z)$

$\zeta_{0}(z)=\sin (z)-i \cos (z)$

$\zeta_{n}(z)=(n-1) \zeta_{n-1}(z) / z-\zeta_{n-2}(z)$

$\psi_{n}(z)=\operatorname{Re}\left\{\zeta_{n}(z)\right\}$

\section{Appendix D: Effective medium theories}

Effective medium theories (EMT) describe the global dielectric function of a composite material from the known dielectric functions of the individual materials that enter into its composition. They are often used in the case one of the material dominates in terms of volume fraction, and in a way "hosts" the others constituents as inclusions. These inclusions must have sizes much less than the wavelength for the local static field approximation leading to these formula to be valid. Many formulae have been obtained following different developments or approximations, relying on distinct shape inclusions, sizes of the inclusions, behaviour at various filling factors, percolation effects, etc. Among these approximation are the Maxwell-Garnett 1904 (for spherical inclusions):

$\epsilon_{\mathrm{eff}}=\epsilon_{m}+3 f_{\mathrm{i}} \epsilon_{m} \frac{\epsilon_{\mathrm{i}}-\epsilon_{m}}{\epsilon_{\mathrm{i}}+2 \epsilon_{m}-f_{\mathrm{i}}\left(\epsilon_{\mathrm{i}}-\epsilon_{m}\right)}$

Brugemann 1925 (symmetrical, for spherical inclusions):

$f_{\mathrm{i}} \frac{\epsilon_{\mathrm{i}}-\epsilon_{\mathrm{eff}}}{\epsilon_{\mathrm{i}}+2 \epsilon_{\mathrm{eff}}}+\left(1-f_{\mathrm{i}}\right) \frac{\epsilon_{m}-\epsilon_{\mathrm{eff}}}{\epsilon_{m}+2 \epsilon_{\mathrm{eff}}}=0$

Looyenga 1965:

$\epsilon_{\mathrm{eff}}^{1 / 3}=f_{\mathrm{i}} \epsilon_{\mathrm{i}}^{1 / 3}+\left(1-f_{\mathrm{i}}\right) \epsilon_{m}^{1 / 3}$

Lichtenecker 1926 (logarithmic mixing case):

$\log \left(\epsilon_{\mathrm{eff}}\right)=f_{\mathrm{i}} \log \left(\epsilon_{\mathrm{i}}\right)+\left(1-f_{\mathrm{i}}\right) \log \left(\epsilon_{m}\right)$

where $f_{\mathrm{i}}$ is the fractional volume of inclusions with respect to the total volume, $\epsilon_{\mathrm{i}}, \epsilon_{\mathrm{m}}$ and $\epsilon_{\mathrm{eff}}$ are the complex dielectric functions of the inclusions, the host medium and the resulting effective medium one, respectively. Other hypothese like the coherent potential approximation, Birchak formula and many other developments for particular systems could in principle also be used.

In astrophysics the first two of the above cited EMTs are mainly used. The Maxwell-Garnett should however preferentially be used for media in which inclusions are distinguishable, like spherical inclusions, whereas the Bruggeman one describes better an inhomogeneous medium for which we do not have much information. In addition care must be taken to use

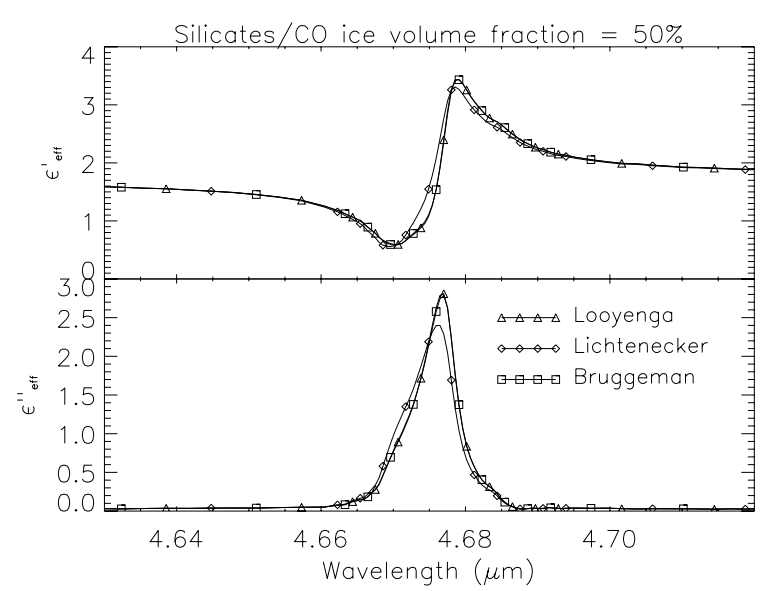

Fig. D.1. Real (upper panel) and imaginary (lower panel) plots of the dielectric functions of a material composed of 50\% silicates and 50\% pure $\mathrm{CO}$ ice, as calculated with the three effective medium theories of Looyenga (triangles), Lichtenecker (diamonds) and Bruggeman (squares). See text for explanation.

the Maxwell-Garnett equation for inclusions that do not exceed $\sim 15 \%$ of the total volume, because by definition this approximation is not symmetrical. In a $50 \%$ repartition of the volumes, the exchange of matrix versus inclusion dielectric functions in the formula leads to two different effective dielectric function. As an example, we have displayed the results of the Looyenga, Lichtenecker and Bruggeman effective medium resultant dielectric functions for a mixture of a silicates (with $\epsilon^{\prime}=2.721$ and $\epsilon^{\prime \prime}=0.132$, constant over the wavelength range for simplicity) and the dielectric function of pure $\mathrm{CO}$ ice shown in Fig. 5. The difference for the Looyenga and Bruggeman EMTs is less than $2 \%$ over the covered range, whereas the Lichtenecker EMT deviates by up to $25 \%$ in the profile wings with respect to the two other EMTs. 